\title{
Robotic Manipulation for Specialty Crop Harvesting: A Review of Manipulator and End-Effector Technologies
}

\author{
Joseph Davidson ${ }^{a, b}$, Santosh Bhusal ${ }^{c, d}$, Changki Mo ${ }^{a, d}$, Manoj Karkee ${ }^{c, d^{*}}$, and Qin Zhang ${ }^{c, d}$ \\ ${ }^{a}$ School of Mechanical and Materials Engineering, Washington State University, TriCities, Washington, United States \\ ${ }^{b}$ Currently in School of Mechanical, Industrial \& Manufacturing Engineering, Oregon State University, Corvallis, Oregon, United States \\ ${ }^{c}$ Biological Systems Engineering Department, Prosser, Washington State University, Prosser, Washington, United States \\ ${ }^{d}$ Center for Precision and Automated Agricultural Systems (CPAAS), Washington State University, Prosser, Washington, United States
}

\section{MANUSCRIPT INFO}

Article history:

Received 26 May 2019

Received in revised form 28 July 2020

Accepted 30 July 2020

Keywords:

manipulator

end-effector

mechanical harvesting

specialty crops

\begin{abstract}
A B S T R A C T
This paper reviews recent developments in manipulator and end-effector technologies for the robotic harvesting of specialty crops that include fruits, vegetables, nursery crops, and nuts among others. Quantitative performance measures and general review criteria, including methods of crop detachment and end-effector sensing, are used to evaluate technologies and determine state-of-the-art in the field. Challenges affecting commercial implementation, limitations of current mechanical designs, and best practices are then presented. Results of the review show that, in general, robotic manipulation during harvesting has been limited by lack of system optimization and insufficient robustness to position error accumulated during visual localization. Inconsistent reporting practices have also hampered research and development across the field. At the conclusion of the review, some avenues of future research that could potentially lead to improvements in system performance are proposed. Some of the proposed recommendations include specific horticultural practices, the development of modular, multi-functional designs, and the incorporation of robust grasping techniques used in many of today's robotic hands.
\end{abstract}

(C) 2020 NAPA. All rights reserved.

\section{Citation:}

Davidson, J., Bhusal, S., Mo, C., Karkee, M., \& Zhang, Q. (2020). Robotic Manipulation for Specialty Crop Harvesting: A Review of Manipulator and End-Effector Technologies. Global Journal of Agricultural and Allied Sciences, 2(1), 25-41.

\section{Introduction}

Over the past two centuries, mechanized agriculture has significantly increased food production efficiency and replaced many jobs previously held by manual labor. However, most advances in agricultural mechanization have been primarily limited to the production of bulk commodity crops like corn and wheat. The production of high-value specialty crops, which the United States Department of Agriculture (USDA) defines as fruits and vegetables, tree nuts, dried fruits, horticulture, and nursery crops (USDA, 2008), is still largely dependent on manual labor. The most time-sensitive and labor-intensive task in specialty crop production is usually harvesting. The physical act of crop harvesting is highly repetitive and physically demanding semi-skilled work, the type of work that has been replaced by automation in other industries (e.g., manufacturing). Increases in specialty crop production yields are needed to accommodate worldwide population growth. Likewise, numerous agricultural sectors around the world, especially those in nations with industrialized economies, also face considerable economic pressure because of rising labor costs and increasing uncertainty about the availability of farm labor. An example of one of these sectors is the fresh market apple industry in Washington State. In 2014, Washington produced 2.7 million metric tons of apples valued at approximately $\$ 1.84$ billion USD (USDA, 2002). The harvest required the seasonal employment of 30,000 additional workers, much of which was supplied by immigrant Latino populations, with an estimated harvest cost of $\$ 1,100$ to $\$ 2,100$ USD per acre per year (Galinato \& Gallardo, 2011; Gallardo, Taylor \& Hinman, 2010). A recent study by the Pew Research Center found that over the past five years net migration from Mexico to the United States has been negative (Gonzalez-Barrera, 2015). The lack of mechanical harvesting technologies for Washington's fresh market apples is a significant problem receiving attention from both federal agencies (e.g., U.S. Department of Agriculture) and state and local organizations (e.g., Washington Tree Fruit Research Commission).

To reduce harvesting costs and the dependence on seasonal labor, researchers have tried two different approaches for specialty crop mechanical harvesting, bulk harvesting with shake-and-catch systems, and selective harvesting with robotics. Shake-and-catch systems (Erdogan, Guner, Dursun \& Gezer, 2003; Peterson, Miller \& Whitney, 1994; Polat et al., 2007) have proven more promising for the mass harvesting of process

* Corresponding author. E-mail address: manoj.karkee@wsu.edu 
market fruit with established tolerances for bruising and external defects. There have also been several attempts to develop mass harvesting systems for fresh market citrus, cherries, and apples (Torregrosa, Orti, Martin, Gil \& Ortiz, 2009). However, these systems either demonstrated marginal rates of fruit detachment (were efficient with only compatible tree-training systems; Peterson, Whiting \& Wolford, 2003), or frequently harvested fruit without stems (Peterson \& Wolford, 2003). Subsequent studies have demonstrated that although the problem can be alleviated with biological control, stem pulls predispose certain apple cultivars to disease (Janisiewicz \& Peterson, 2004).

The use of robotics technology to selectively harvest individual fruit is the second approach researchers have tried for harvesting specialty crops including tree fruits and vegetables. In this article, the term "fruit" is used to refer to the target product regardless if it is a fruit or vegetable. For economic reasons related to changing labor conditions, scientists and engineers started to actively work on research and development of fruitpicking robots in the 1980s (Grand D'Esnon, 1985; Harrell \& Levi, 1988). An early review of fruit-picking robotics research was conducted by Sarig (1993), who evaluated progress on both machine vision technologies used to locate fruit and robotic manipulators that reached for the intended target. Sarig (1993) noted that most experts estimated that robotic fruit-harvesting systems in R\&D would transition to commercial use by the end of the Twentieth Century. Other comprehensive reviews have been completed by Burks et al. (2005), Li, Lee \& Hsu (2011) and Shamshri et al. (2018a). The most recent work was an excellent review of robotic harvesting by Bac, van Henten, Hemming \& Edan (2014) and is an extensive analysis of the crop environment, design methodologies, algorithms, hardware, logistics, and economics. Their analysis used quantitative performance measures to evaluate state-of-the-art harvesting robots and progress in the field over the past 30 years. Despite extensive research and the numerous robotic harvesting projects/technologies undertaken, the authors found little improvement in system performance and no evidence of commercial implementation.

To improve the performance of robotic harvesting systems, Bac et al. (2014) proposed that researchers address two challenges, simplifying the task by modifying the crop environment and enhancing the robot performance. Some of their suggested modifications to the crop environment to improve performance include modified cultivation systems, desired cultivar selection and breeding, and alternative cultivation practices. Regarding robot performance, it is widely recognized that visual sensing and mechanical manipulation are the subsystems requiring the most improvement. While not the subject of this paper, a significant issue facing machine vision researchers is enhancing the system's ability to determine fruit location and ripeness when the target product is obscured by leaves, branches, and other fruits. The specific focus of this review paper, however, is the robotic harvester's manipulator and end-effector performance. Whereas numerous publications review state-of-the-art in vision research (Gongal, Amatya, Karkee, Zhang \& Lewis, 2015; Jimenez, Ceres \& Pons, 2000; Kapach, Barnea, Mairon, Edan \& Ben-Shahar, 2012), a gap in the current literature makes it difficult to adequately assess the status of mechanical manipulation during the robotic harvesting of specialty crops. A recent review (Rodriguez, Moreno, Sanchez \& Berenguel, 2013) used the Cutkosky taxonomy (Cutkosky, 1989) to classify agricultural end-effectors, but this review was limited to grasping end-effectors only. This paper attempts to extend the understanding of robot performance through a detailed and comprehensive analysis of the mechanical design of both the manipulator and harvesting end-effector.

Section 2 of this paper provides a brief summary of the system requirements as well as the crop environmental parameters that are particularly relevant to the mechanical design of the manipulator and endeffector. While the parameters in the agricultural environment that affect vision, like variable lighting and occlusion, are well-documented, less attention has been paid to the considerations influencing mechanical design decisions. Because the focus of this article is the manipulator and endeffector, environmental variables that impact mobile platforms used to move the manipulator are not considered. Section 3 presents the method used in reviewing the literature as well as the indicators used to measure performance. Results of the literature review are summarized and then discussed in Sections 4 and 5, respectively. In Section 6, the authors propose some possible avenues of future research for improving the performance of harvesting end-effectors. Because the fresh market apple industry is an agricultural sector with significant potential for the incorporation of robotic harvesting technologies and is also an area of research of interest to the authors, factors and considerations that are particularly applicable to apple or similar fruit picking are highlighted. Additionally, because developments in manipulator and end-effector technologies are often cross-cutting, all specialty crop harvesting applications are considered. The technologies and systems reviewed have been designed for field, orchard, and greenhouse applications (Shamshiri et al., 2018b).

\section{System Requirements}

The highly unstructured agricultural field/orchard poses unique engineering challenges compared to the orderly manufacturing lines of industrial factories where automation and robotics technologies have augmented or altogether replaced manual labor. This section presents the functionality needed by harvesting robots and then discusses how the unstructured agricultural environment guides the selection of certain design criteria. The basic functional requirements of a harvesting robot include the following general sequence of tasks: 1. Detection of the fruit in the scene, identification of its properties (e.g. level of ripeness), and localization of the fruit in 3-dimensional space, 2. Approach to target fruit, 3. Detachment of the fruit from the plant, and 4. Guiding the harvested product to the storage container. Task 1 is typically accomplished with the use of a machine vision system. Our focus in this review is analysis of performance against functional requirements two through four.

\subsection{Tasks 2 \& 4: Approach to the Fruit and Guiding the Harvested Product to the Storage Container}

For selective harvesting of specialty crops, a robotic manipulator will guide the end-effector to the target fruit and then deposit the harvested product in a storage container. The harvesting robot must be able to successfully navigate the crop environment while minimizing damage to the plant. Therefore, an essential design criterion of the manipulator is a kinematic framework flexible enough to accommodate the crop environment. The workspace required is highly dependent on environmental parameters like 
plant spacing, crop height, and fruit distribution, and should be defined early in the design process. As an example of how the environment can influence manipulator design choices, consider the two types of apple orchards shown in Figure 1. The orchard shown on the left uses a modern cultivation system where the trees are supported by a wire and post trellis system. This two-dimensional, planer canopy produces a "fruit wall" designed to increase productivity by enhancing visibility and accessibility of the fruit. The orchard shown on the right uses a conventional cultivation system that produces fuller tree canopies whereby the fruit are in three dimensions. It is expected that the kinematic design and motion planning/obstacle avoidance requirements would be less rigorous for the harvesting robot operating in the modern apple cultivation environment.
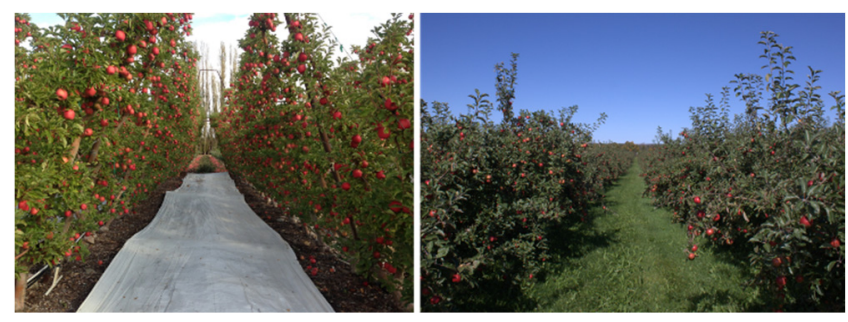

Figure 1. Apple orchards. The orchard at left is a well-maintained, modern orchard using the $V$-trellis cultivation system. The orchard shown on the right is an older orchard with 3-dimensional tree canopies.

A second design criteria for the manipulator is a model dynamic enough to sustain the product's payload and apply the necessary fruit detachment forces, if any. For example, a harvesting robot picking watermelons will have different transmission and actuation requirements than a system harvesting mushrooms. The selection of the manipulator design should occur parallel with the design of the end-effector. If the end-effector cuts the stem of the fruit, the dynamic loading on the manipulator would be less compared to a system that uses an end-effector to grasp the fruit and the manipulator to apply a certain sequence of detachment motions.

Another design criterion is that the system must be capable of continuous operations in its agricultural environment, which could be an open-field or closed-field operation. Robots working in outdoor environments will be exposed to harsher operating conditions. Actuators, sensors, and control systems may need to be protected against rain, wind, dust, and large temperature fluctuations. Additionally, power sources for electric motors, compressors, and hydraulic pumps are more likely to be accessible in indoor environments. Robotic harvesters working in outdoor fields and orchards may also require an external generator.

An additional and fundamental design criterion for harvesting robot prototypes is economic feasibility. However, because the focus in this review is mechanical manipulation performance during harvesting, economic feasibility is not a parameter used to evaluate state-of-the-art in this field. It is difficult to assess and is highly dependent on the market and crop location. However, mechanical performance measured through the contexts of picking times and product damage rates is a major consideration during evaluation of the system's overall economic feasibility. Also, depending on the terrain and labor support available, a relatively robust and simple design could potentially be another system requirement. Because agricultural technicians responsible for maintaining the equipment may not possess the same level of robot-specific training as industrial robotics technicians, maintenance requirements and reliability impact cost evaluations.

\subsection{Task 3: Fruit Detachment}

The end-effector is the tool attached to the end of the manipulator that removes the fruit from the plant and is, therefore, the system component responsible for functional requirement of Task 3 . In addition to navigating complex, irregular plant structures, the harvesting robot and its end-effector must also be capable of handling a high degree of variability that exists in the targeted crop. Karkee and Zhang (2012) and Bac et al. (2014) have discussed several sources of variation of objects in a crop. Due to numerous environmental factors, objects in a crop exhibit natural variation in fruit position, shape, size, growing orientation, and stem length. The level of variation depends to a large degree on the crop and growing environment.

Even for the same cultivar, parameters such as fruit color, size, and stem length vary widely within a tree. Figure 2 shows two pictures of apples growing in the same tree. The fruit shown on the left are isolated from other fruits, have exposed stems, grow at the end of branches in vertical orientations, and are in general more accessible. However, the fruit on the right are clustered, are oriented in unpredictable directions, tend to be occluded by branches and other fruits, and are in general less accessible. These parameters related to fruit growth patterns are important considerations in the design of the end-effector and its method of fruit detachment. Growth characteristics play a significant role in the design choice on manipulation strategy. Because of the high degree of inconsistency between individual fruits, end-effector robustness to object shape, size, position, and orientation may be critical design criteria.
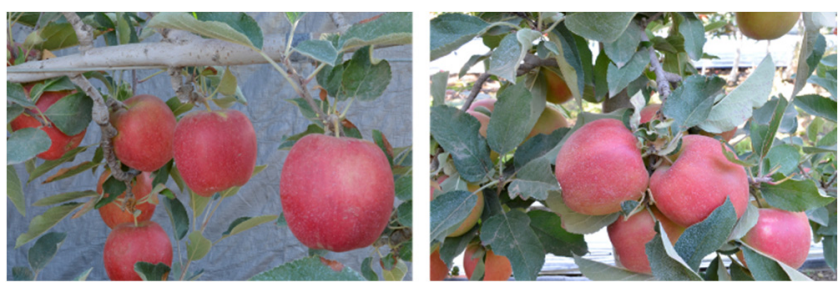

Figure 2. Variation in apple fruiting position and orientation.

A second end-effector design criteria is that it should minimize damage to both the plant and the harvested fruit to a tolerable level. To be acceptable for the fresh market specialty crops must be free of bruising, incisions, and other external defects. Because of the highly delicate nature of many of the products (e.g., apples, berries, cherries, mushrooms), it is important that understanding of the fruit's physical properties guides the selection of the fruit removal technique and the overall end-effector design. For example, a design that grasps the fruit should not produce forces that exceed the threshold that causes bruising. Some of the fruit's relevant physical properties include friction of the fruit's surface, mass, firmness, and tensile strength/cutting resistance of the fruit's stem. 


\section{Method of Review}

The authors have completed a literature review of fruit harvesting robotics technologies with results published in English-language conferences and journal papers. This area of research is highly interdisciplinary with results distributed across numerous types of publications (e.g., field robotics, agricultural engineering, and horticultural practices). Consolidating and analyzing the results from these different forums was challenging. Only papers that provided a thorough description of the manipulator and endeffector were considered. Those papers primarily dedicated to discussion of the machine vision system are not presented. The technologies evaluated included the robotic harvesting of specialty crops in all production environments (e.g., field, orchard, and greenhouse). Our goal was to determine how design selections to address the functional requirements related to mechanical manipulation influenced overall harvesting robot performance. The relationship between performance characteristics against design choices such as fruit detachment method and sensor selection was analyzed in order to determine current challenges, positive trends, and best practices. A description of the quantitative performance measures and general criteria used to assess a project follows.

\subsection{Performance Indicators}

Three quantitative performance indicators were selected to assess the technologies. The first indicator used is picking time(s). This indicator marks the time elapsed from the completion of fruit localization to the detachment and storage of the fruit. Note, this indicator is different from the cycle time(s) as defined by Bac et al. (2014), which includes the time required for ripeness determination and fruit localization. By removing the portion of time required for vision processing the picking time indicator provides a specific measure of manipulator and end-effector speed. The other two performance indicators included are detachment success (\%), which is the percentage of successfully detached fruit per total fruit localized in the robot's workspace and damage rate (\%). As noted in the introduction, an apple stem-pull is considered damage because it predisposes certain cultivars to disease. Detachment of the fruit spur or other plant components that would remove a fruit position from the next year's crop is also considered damage. Detachment success and damage rate are highly relevant for assessment of manipulation performance.

\subsection{Application of Design Method}

During the review it was noted whether a formal design process that included analysis of functional requirements and design specifications was applied during system development. Efforts to characterize the crop workspace and optimization of manipulator and end-effector design parameters in accordance with this workspace were considered in the application of design methodology. The implementation of crop cultivation systems specifically developed to enhance the feasibility of automated harvesting was also considered evidence of the application of a formal design process.

We also examined whether end-effector design choices were supported by data on the physical properties of the target fruit. Characterization of these properties is an important tool when defining constraints during the fruit removal process. Technologies were reviewed to see if they conducted their own studies or referenced already available data. Numerous studies on fruit physical properties have already been discussed in the literature. For example, to support the development of robotic harvesting Li, Li \& Liu (2011) have published results from tests designed to investigate the physical properties of tomatoes relevant to robotic harvesting. Some of the properties measured during tests included friction coefficients, rupture force, and compressibility. Another project on the physical properties of tomatoes has been completed by Guo, Zhan, Ping \& Jun (2009). Likewise, Flood, Burks \& Teixeira (2006) conducted compressive force testing on oranges using both punch tests and burst tests in order to make recommendations on the design of a grasping robotic citrus harvester endeffector.

\subsection{Manipulator Kinematics and Actuator Selection}

Manipulator design was reviewed in terms of the actuators used (i.e., electric, hydraulic, or pneumatic), degree of freedom (DOF) selected, and whether it was a custom design. It was also noted whether on-line collision detection and obstacle avoidance was included during the picking process.

\subsection{Method of Fruit Detachment}

The results of the review (Section 4) will show that many different fruit removal techniques, including gripping and cutting, have been incorporated in end-effector designs. We list whether the end-effector contacts the fruit, the peduncle (stem), or both, and classify the type of manipulation strategy adopted. Gripping is defined as a single DOF method applied with vacuum or open/close devices. Grasping describes a manipulation scheme with multiple DOF that is adaptive to the product's shape. Cutting of the stem is accomplished with a blade or thermal device. To ascertain the level of complexity used in the design, we reviewed whether in-device manipulation of the harvested fruit was used. It was also noted if the endeffector completed all fruit removal steps, or if manipulator motions/dynamic loads were required to assist with fruit detachment. The purpose of reviewing these parameters was to determine if trends exist between certain removal methods and end-effector performance and robustness.

\subsection{End-Effector Actuator and Sensor Selection}

During our review we were also very interested whether sensors were used to control the end-effector. If sensors were incorporated in the design, the number and type (e.g., force and proximity) used were listed. If a camera was mounted on the end-effector to assist with guidance and visual serving, it was classified as an end-effector sensor.

\section{Results}

The authors have reviewed 39 different robotic harvesting technologies completed between the years 1985 and 2018. Results of the review are presented in a concise form in Table 1, which was adapted from Bac et al. (2014). All technologies reviewed were developed for fruit or vegetable harvesting. If we inferred data from a reviewed project, we placed a question mark beside the table entry to indicate some level of uncertainty. 
Table 1. Agricultural Harvesting Robotics Technologies: 1986 - 2012.

\begin{tabular}{|c|c|c|c|c|c|c|c|c|c|c|c|c|c|c|c|c|c|c|}
\hline \multirow[b]{2}{*}{ Fruit } & \multirow[b]{2}{*}{ Study } & \multirow[b]{2}{*}{$\begin{array}{l}\text { Design } \\
\text { Proces } \\
\quad \mathrm{s}\end{array}$} & \multicolumn{3}{|c|}{ Manipulator } & \multicolumn{2}{|c|}{$\begin{array}{l}\text { Contact } \\
\text { Points }\end{array}$} & \multicolumn{4}{|c|}{$\begin{array}{c}\text { Fruit Detachment } \\
\text { Method }\end{array}$} & \multicolumn{2}{|c|}{ End-Effector Actuators } & \multicolumn{2}{|c|}{$\begin{array}{c}\text { End-Effector } \\
\text { Sensors }\end{array}$} & \multirow[b]{2}{*}{$\begin{array}{c}\text { Picki } \\
\text { ng } \\
\text { Time } \\
*\end{array}$} & \multirow{2}{*}{$\begin{array}{c}\text { Detach- } \\
\text { ment } \\
\text { Success } \\
\text { Rate* }\end{array}$} & \multirow[b]{2}{*}{ Damage Rate* } \\
\hline & & & ํํำ & 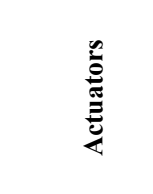 & 这 & 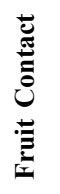 & 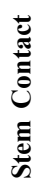 & ํํㄹ & ज़ & $\Xi$ & 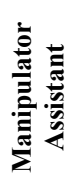 & $\#$ & $\overbrace{i}$ & $\#$ & $\overbrace{2}$ & & & \\
\hline Apple & $\begin{array}{l}\text { Grand d'Esnon et } \\
\text { al., } 1987\end{array}$ & - & 4 & Hydraulic & - & $\checkmark$ & - & $\checkmark$ & - & - & $\checkmark$ & 1 & Vacuum & - & - & $4 \mathrm{~s}(?)$ & $50.0 \%$ & $25 \%$ \\
\hline Apple & $\begin{array}{c}\text { Setiawan, } \\
\text { Furukawa, \& } \\
\text { Preston, } 2004\end{array}$ & - & 6 & $\begin{array}{l}\text { Electric } \\
\text { (industrial) }\end{array}$ & - & $\checkmark$ & - & $\checkmark$ & - & - & $\checkmark$ & 1 & $\begin{array}{l}\text { Vacuum } \\
\text { generator }\end{array}$ & 0 & - & $11.9 \mathrm{~s}$ & - & - \\
\hline Apple & $\begin{array}{l}\text { Baeten et al., } \\
2008\end{array}$ & - & 6 & $\begin{array}{l}\text { Electric } \\
\text { (industrial) }\end{array}$ & - & $\checkmark$ & - & $\checkmark$ & - & - & $\checkmark$ & 1 & Vacuum pump & 1 & Camera & $8 \mathrm{~s}(?)$ & $80.0 \%$ & $30 \%$ \\
\hline Apple & $\begin{array}{c}\text { Bulanon \& } \\
\text { Kataoka, } 2010\end{array}$ & - & 3 & $\begin{array}{l}\text { Electric? } \\
\text { (custom) }\end{array}$ & - & - & $\checkmark$ & $\checkmark$ & - & - & $\checkmark$ & 2 & $\begin{array}{l}\text { DC motor \& } \\
\text { stepper motor }\end{array}$ & 0 & - & $7.1 \mathrm{~s}$ & $90.9 \%$ & - \\
\hline Apple & Zhao et al., 2011 & - & 5 & $\begin{array}{l}\text { Electric \& } \\
\text { Hydraulic? } \\
\text { (custom) }\end{array}$ & $\checkmark$ & $\checkmark$ & $\checkmark$ & $\checkmark$ & - & $\checkmark$ & - & 2 & $\begin{array}{l}\text { DC Motor \& } \\
\text { pneumatic } \\
\text { pump }\end{array}$ & 4 & $\begin{array}{l}\text { Collision, } \\
\text { pressure, } \\
\text { position, } \\
\& \text { vision }\end{array}$ & $\begin{array}{c}13 \mathrm{~s} \\
(?)\end{array}$ & $77.0 \%$ & - \\
\hline Apple & Silwal et al., 2017 & $\checkmark$ & 7 & $\begin{array}{l}\text { Electric } \\
\text { (custom) }\end{array}$ & - & $\checkmark$ & - & - & $\checkmark$ & - & - & 3 & Electric & 0 & - & $6.0 \mathrm{~s}$ & $84.6 \%$ & 0 \\
\hline Apple & $\begin{array}{l}\text { Hohimer et al., } \\
2018\end{array}$ & & 5 & $\begin{array}{l}\text { Electric } \\
\text { (custom) }\end{array}$ & - & $\checkmark$ & - & - & $\checkmark$ & - & - & 3 & Pneumatic & 0 & - & - & $67.0 \%$ & \\
\hline Asparagus & Irie et al, 2009 & - & 4 & $\begin{array}{l}\text { Electric } \\
\text { (custom) }\end{array}$ & - & $\checkmark$ & $\checkmark$ & $\checkmark$ & - & $\checkmark$ & - & 2 & Electric motor & - & - & $8.7 \mathrm{~s}$ & - & - \\
\hline Asparagus & $\begin{array}{l}\text { Chatzimichali et } \\
\text { al., } 2009\end{array}$ & - & $\begin{array}{c}3 \\
(?)\end{array}$ & $\begin{array}{l}\text { Electric } \\
\text { (custom) }\end{array}$ & - & $\checkmark$ & $\checkmark$ & $\checkmark$ & - & $\checkmark$ & - & 2 & $\begin{array}{l}\text { Pneumatic } \\
\text { pump \& DC } \\
\text { motor }\end{array}$ & - & - & - & - & - \\
\hline Cantaloupe & Edan et al., 2000 & $\checkmark$ & 3 & $\begin{array}{l}\text { Electric? } \\
\text { (custom) }\end{array}$ & - & $\checkmark$ & $\checkmark$ & $\checkmark$ & - & $\checkmark$ & - & 2 & Pneumatic (?) & 2 & $\begin{array}{l}\text { Proximity } \\
\& \text { vision }\end{array}$ & $15 \mathrm{~s}$ & $85.0 \%$ & $7 \%$ \\
\hline Cherry & $\begin{array}{l}\text { Tanigaki et al., } \\
2008\end{array}$ & - & 4 & $\begin{array}{l}\text { Electric } \\
\text { (custom) }\end{array}$ & $\checkmark$ & $\checkmark$ & $\checkmark$ & $\checkmark$ & - & - & $\checkmark$ & 3 & $\begin{array}{l}\text { Servomotors } \\
\& \text { vacuum } \\
\text { pump }\end{array}$ & 1 & Pressure & $\begin{array}{c}14 \mathrm{~s} \\
(?)\end{array}$ & $78.0 \%$ & $43 \%$ \\
\hline Cucumber & $\begin{array}{l}\text { Van Henten et } \\
\text { al., } 2002\end{array}$ & $\checkmark$ & 7 & $\begin{array}{l}\text { Electric } \\
\text { (industrial) }\end{array}$ & $\checkmark$ & $\checkmark$ & $\checkmark$ & $\checkmark$ & - & $\checkmark$ & - & 2 & $\begin{array}{l}\text { Pneumatic } \\
\text { pump \& } \\
\text { electric motor }\end{array}$ & 1 & Camera & - & $80.0 \%$ & - \\
\hline Cucumber & Tang et al., 2009 & $\checkmark$ & 6 & $\begin{array}{l}\text { Electric } \\
\text { (custom) }\end{array}$ & - & - & $\checkmark$ & - & - & $\checkmark$ & - & 1 & Electric motor & 0 & - & - & $93 \%(?)$ & - \\
\hline
\end{tabular}




\begin{tabular}{|c|c|c|c|c|c|c|c|c|c|c|c|c|c|c|c|c|c|c|}
\hline \multirow[b]{2}{*}{ Fruit } & \multirow[b]{2}{*}{ Study } & \multirow[b]{2}{*}{$\begin{array}{l}\text { Design } \\
\text { Proces } \\
\quad \mathrm{s}\end{array}$} & \multicolumn{3}{|c|}{ Manipulator } & \multicolumn{2}{|c|}{$\begin{array}{c}\text { Contact } \\
\text { Points } \\
\end{array}$} & \multicolumn{4}{|c|}{$\begin{array}{c}\text { Fruit Detachment } \\
\text { Method }\end{array}$} & \multicolumn{2}{|c|}{$\begin{array}{c}\text { End-Effector } \\
\text { Actuators }\end{array}$} & \multicolumn{2}{|c|}{$\begin{array}{c}\text { End-Effector } \\
\text { Sensors }\end{array}$} & \multirow[b]{2}{*}{$\begin{array}{l}\text { Picking } \\
\text { Time* }\end{array}$} & \multirow{2}{*}{$\begin{array}{c}\text { Detach- } \\
\text { ment } \\
\text { Success } \\
\text { Rate* }\end{array}$} & \multirow[b]{2}{*}{ Damage Rate* } \\
\hline & & & ำ & $\frac{n}{\tilde{\omega}}$ & 泀 & 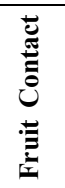 & 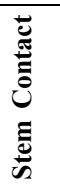 & 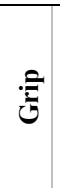 & जै & $\Xi$ & 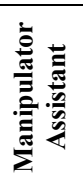 & $\#$ & $\sum_{i=1}^{\circ}$ & $\#$ & $\sum_{=}^{\circ}$ & & & \\
\hline Date Palm & $\begin{array}{c}\text { Aljanobi et al., } \\
2010\end{array}$ & - & 5 & $\begin{array}{l}\text { Electric } \\
\text { (custom) }\end{array}$ & - & $\checkmark$ & - & $\checkmark$ & - & - & $\checkmark$ & 1 (?) & Motor (?) & - & - & - & - & - \\
\hline Eggplant & $\begin{array}{l}\text { Wan Ishak, Kit, } \\
\text { \& Awal, } 2010\end{array}$ & - & 3 & $\begin{array}{l}\text { Electric } \\
\text { (custom) }\end{array}$ & - & $\checkmark$ & $\checkmark$ & - & - & $\checkmark$ & - & 1 & DC Motor & 0 & - & - & - & - \\
\hline Grape & $\begin{array}{l}\text { Monta, Kondo, } \\
\text { \& Shibano, } 1995\end{array}$ & - & 5 & $\begin{array}{l}\text { Electric? } \\
\text { (custom) }\end{array}$ & - & - & $\checkmark$ & $\checkmark$ & - & $\checkmark$ & - & $?$ & $?$ & 1 & Camera & - & - & - \\
\hline Kiwi & $\begin{array}{l}\text { Scarfe et al., } \\
2009\end{array}$ & $\checkmark$ & 3 & $\begin{array}{l}\text { Electric } \\
\text { (custom) }\end{array}$ & - & $\checkmark$ & - & $\checkmark$ & - & - & $\checkmark$ & 1 (?) & Electric motor & 0 & - & $1 \mathrm{~s}(?)$ & - & - \\
\hline Lettuce & Cho et al., 2002 & - & 3 & $\begin{array}{l}\text { Electric \& } \\
\text { Pneumatic } \\
\text { (custom) }\end{array}$ & - & $\checkmark$ & $\checkmark$ & $\checkmark$ & - & $\checkmark$ & - & 1 (?) & Pneumatic & - & $\begin{array}{l}\text { Force } \\
\text { control } \\
\text { w/fuzzy } \\
\text { logic }\end{array}$ & $5 \mathrm{~s}(?)$ & $94.0 \%$ & $6 \%$ \\
\hline Lychee & Liu et al., 2011 & - & - & - & - & - & $\checkmark$ & $\checkmark$ & - & $\checkmark$ & - & 1 & Pneumatic & - & - & - & - & - \\
\hline Mushrooms & Reed et al., 2001 & - & 3 & $\begin{array}{l}\text { Pneumatic } \\
\text { (custom) }\end{array}$ & - & $\checkmark$ & - & $\checkmark$ & - & - & - & 3 & $\begin{array}{c}\text { Stepper motor } \\
\& \text { rotary } \\
\text { pneumatic } \\
\text { actuators }\end{array}$ & 0 & - & $\begin{array}{c}6.7 \mathrm{~s} \\
(?)\end{array}$ & $86.0 \%$ & $3 \%$ \\
\hline Orange & $\begin{array}{l}\text { Pool and Harrell, } \\
1991\end{array}$ & - & 3 & $\begin{array}{l}\text { Hydraulic } \\
\text { (custom) }\end{array}$ & - & $\checkmark$ & $\checkmark$ & $\checkmark$ & - & - & $\checkmark$ & 1 & Hydraulic & 2 & $\begin{array}{c}\text { CCD } \\
\text { camera and } \\
\text { ultrasonic } \\
\text { transducer }\end{array}$ & $\begin{array}{c}3-7 \mathrm{~s} \\
(?)\end{array}$ & $69.0 \%$ & $44 \%$ \\
\hline Orange & $\begin{array}{l}\text { Muscato et al., } \\
2005\end{array}$ & - & 3 & $\begin{array}{l}\text { Electric \& } \\
\text { Pneumatic } \\
\text { (custom) }\end{array}$ & - & $\checkmark$ & $\checkmark$ & - & - & $\checkmark$ & - & 3 & Pneumatic & 2 & $\begin{array}{l}\text { proximity } \\
\text { sensor } \\
\& \text { camera }\end{array}$ & $8.7 \mathrm{~s}$ & - & - \\
\hline Orange & $\begin{array}{l}\text { Lee and Rosa, } \\
2006\end{array}$ & - & 3 & $\begin{array}{l}\text { Hydraulic \& } \\
\text { pneumatic } \\
\text { (custom) }\end{array}$ & - & - & $\checkmark$ & - & - & $\checkmark$ & - & 1 & $\begin{array}{l}\text { Pneumatic } \\
\text { cylinder }\end{array}$ & 0 & - & - & $84.0 \%$ & - \\
\hline Pineapple & $\begin{array}{l}\text { Haifeng et al, } \\
2002\end{array}$ & - & - & - & - & $\checkmark$ & - & $\checkmark$ & - & - & $\checkmark$ & 2 & Stepper motors & $1(?)$ & Force & $23 \mathrm{~s}$ & - & - \\
\hline Radicchio & $\begin{array}{l}\text { Foglia and Reina, } \\
2006\end{array}$ & $\checkmark$ & 2 & $\begin{array}{l}\text { Pneumatic } \\
\text { (custom) }\end{array}$ & - & $\checkmark$ & $\checkmark$ & $\checkmark$ & - & $\checkmark$ & - & 2 & $\begin{array}{l}\text { Pneumatic } \\
\text { muscles }\end{array}$ & 4 & $\begin{array}{c}\text { Limit } \\
\text { switches }\end{array}$ & $6.5 \mathrm{~s}$ & - & - \\
\hline $\begin{array}{l}\text { Cherry } \\
\text { Tomato }\end{array}$ & $\begin{array}{c}\text { Kondo et al., } \\
1996\end{array}$ & $\checkmark$ & 7 & $\begin{array}{l}\text { Electric? } \\
\text { (custom) }\end{array}$ & - & $\checkmark$ & $\checkmark$ & $\checkmark$ & - & $\checkmark$ & - & 2 & $\begin{array}{l}\text { Pneumatic } \\
\& \text { solenoid }\end{array}$ & 3 & $\begin{array}{l}\text { Photo } \\
\text { sensors }\end{array}$ & $2 \mathrm{~s}$ & $70.0 \%$ & - \\
\hline Tomato & $\begin{array}{l}\text { Monta et al., } \\
1998\end{array}$ & $\checkmark$ & 7 & $\begin{array}{l}\text { Electric } \\
\text { (custom) }\end{array}$ & - & $\checkmark$ & $\checkmark$ & $\checkmark$ & - & - & $\checkmark$ & 2 & $\begin{array}{l}\text { DC motor } \\
\& \text { vacuum } \\
\text { pump }\end{array}$ & 2 & $\begin{array}{l}\text { Potentiomet } \\
\text { er } \\
\& \text { pressure }\end{array}$ & $15 \mathrm{~s}$ & $91.0 \%$ & - \\
\hline
\end{tabular}




\begin{tabular}{|c|c|c|c|c|c|c|c|c|c|c|c|c|c|c|c|c|c|c|}
\hline \multirow[b]{2}{*}{ Fruit } & \multirow[b]{2}{*}{ Study } & \multirow[b]{2}{*}{$\begin{array}{l}\text { Design } \\
\text { Proces } \\
\quad \mathbf{s}\end{array}$} & \multicolumn{3}{|c|}{ Manipulator } & \multicolumn{2}{|c|}{$\begin{array}{l}\text { Contact } \\
\text { Points } \\
\end{array}$} & \multicolumn{4}{|c|}{$\begin{array}{c}\text { Fruit Detachment } \\
\text { Method }\end{array}$} & \multicolumn{2}{|c|}{$\begin{array}{c}\text { End-Effector } \\
\text { Actuators }\end{array}$} & \multicolumn{2}{|c|}{$\begin{array}{c}\text { End-Effector } \\
\text { Sensors }\end{array}$} & \multirow[b]{2}{*}{$\begin{array}{c}\text { Picking } \\
\text { Time** }\end{array}$} & \multirow{2}{*}{$\begin{array}{c}\text { Detach- } \\
\text { ment } \\
\text { Success } \\
\text { Rate* }\end{array}$} & \multirow[b]{2}{*}{ Damage Rate* } \\
\hline & & & 资 & 总 & لّ & 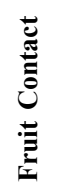 & 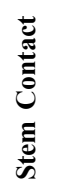 & 吾 & जै & $\Xi$ & 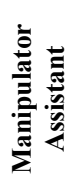 & $\#$ & $\sum_{i=1}^{\circ}$ & $\#$ & ڤ્. & & & \\
\hline Tomato & Ling et al., 2004 & - & 6 & $\begin{array}{c}\text { Electric } \\
\text { (industrial) }\end{array}$ & - & $\checkmark$ & - & $\checkmark$ & $\checkmark$ & - & $\checkmark$ & 2 & $\begin{array}{l}\text { Vacuum pump } \\
\& \text { stepper } \\
\text { motor linear } \\
\text { actuator }\end{array}$ & 1 & $\begin{array}{l}\text { Pressure } \\
\text { transducer }\end{array}$ & - & - & - \\
\hline Tomato & Zhao et al., 2016 & - & 3 & - & - & $\checkmark$ & $\checkmark$ & - & $\checkmark$ & $\checkmark$ & - & 2(?) & Pneumatic (?) & - & & - & - & - \\
\hline Saffron & $\begin{array}{l}\text { Antonelli et al., } \\
2011\end{array}$ & - & 3 & $\begin{array}{l}\text { Electric } \\
\text { (custom) }\end{array}$ & - & - & $\checkmark$ & $\checkmark$ & - & - & $\checkmark$ & 3 & $\begin{array}{l}\text { EM solenoids } \\
\& \text { vacuum } \\
\text { pump }\end{array}$ & 1 & $\begin{array}{l}\text { Optical } \\
\text { sensor }\end{array}$ & - & $60.0 \%$ & - \\
\hline $\begin{array}{l}\text { Spherical } \\
\text { Fruit }\end{array}$ & Liu et al., 2007 & - & 6 & $\begin{array}{l}\text { Electric } \\
\text { (industrial) }\end{array}$ & - & $\checkmark$ & - & $\checkmark$ & - & $\checkmark$ & - & 3 & $\begin{array}{l}\text { DC servomotors } \\
\& \text { vacuum } \\
\text { pump }\end{array}$ & 8 & $\begin{array}{l}\text { Vacuum, } \\
\text { distance, } \\
\text { proximity, } \\
\text { \& force }\end{array}$ & - & - & - \\
\hline Strawberry & $\begin{array}{c}\text { Hayashi et al. } \\
2010\end{array}$ & - & 3 & $\begin{array}{l}\text { Electric? } \\
\text { (custom) }\end{array}$ & - & $\checkmark$ & $\checkmark$ & $\checkmark$ & - & $\checkmark$ & - & 2 (?) & Pneumatic & 1 & $\begin{array}{c}\text { Photoelectri } \\
\mathrm{c}\end{array}$ & $\begin{array}{c}10.5 \mathrm{~s} \\
(?)\end{array}$ & $85.0 \%$ & - \\
\hline Strawberry & Feng et al., 2012 & - & 6 & $\begin{array}{c}\text { Electric } \\
\text { (industrial) }\end{array}$ & - & $\checkmark$ & $\checkmark$ & $\checkmark$ & - & $\checkmark$ & - & 1 (?) & Pneumatic & 0 & - & $\begin{array}{c}31.3 \mathrm{~s} \\
(?)\end{array}$ & $86.0 \%$ & - \\
\hline Strawberry & Han et al., 2012 & - & 4 & $\begin{array}{l}\text { Electric } \\
\text { (custom) }\end{array}$ & - & - & $\checkmark$ & $\checkmark$ & - & $\checkmark$ & - & 4 & Electric motors & 1 & $\begin{array}{l}\mathrm{CCD} \\
\text { camera }\end{array}$ & - & - & - \\
\hline Sweet Pepper & $\begin{array}{l}\text { Kitamura and } \\
\text { Oka, } 2005\end{array}$ & - & - & $\begin{array}{l}\text { Electric } \\
\text { (custom) }\end{array}$ & - & - & $\checkmark$ & - & - & $\checkmark$ & - & 1 & DC motor & 0 & - & - & - & - \\
\hline $\begin{array}{l}\text { Various types } \\
\text { of Fruit } \\
\text { (Universal } \\
\text { end-effector) }\end{array}$ & Jia et al., 2009 & - & 5 & $\begin{array}{c}\text { Electric } \\
\text { (industrial) }\end{array}$ & - & - & $\checkmark$ & $\checkmark$ & - & $\checkmark$ & - & 1 & DC motor (?) & 0 & - & $37 \mathrm{~s}(?)$ & - & - \\
\hline Watermelon & $\begin{array}{l}\text { Umeda, Kubota, } \\
\text { \& Iida, } 1999\end{array}$ & - & 3 & $\begin{array}{l}\text { Electric } \\
\text { (custom) }\end{array}$ & - & $\checkmark$ & - & $\checkmark$ & - & - & $\checkmark$ & 1 & Vacuum pump & - & - & - & $66.0 \%$ & - \\
\hline Watermelon & $\begin{array}{l}\text { Hwang \& Kim, } \\
2003\end{array}$ & - & 4 & $\begin{array}{l}\text { Electric } \\
\text { (custom) }\end{array}$ & - & $\checkmark$ & $\checkmark$ & $\checkmark$ & - & $\checkmark$ & - & 1 (?) & Pneumatic & - & - & $14 \mathrm{~s}(?)$ & - & - \\
\hline Watermelon & $\begin{array}{l}\text { Sakai et al., } \\
2008\end{array}$ & $\checkmark$ & 4 & $\begin{array}{l}\text { Hydraulic } \\
\text { \& electric } \\
\text { (custom) }\end{array}$ & - & $\checkmark$ & - & $\checkmark$ & - & - & - & 1 & DC motor & - & - & $14 \mathrm{~s}$ & $86.0 \%$ & $0 \%$ \\
\hline
\end{tabular}


Dashes were used to indicate that we could not determine the information from the project's reference.

\subsection{Performance Indicators}

Only eight of the 39 technologies reported damage rates, which varied from 0 to $44 \%$. There was a higher incidence of reporting on detachment success rate $(54 \%$, or 21 out of 39$)$, which varied from $50 \%$ to $94 \%$. The average detachment success rate was $79 \%$.

It was difficult to compare picking times because of inconsistent reporting practices used by the research community, a finding also expressed by Bac et al. (2014). For example, Bulanon \& Kataoka (2010) reported that the picking time reflected the time required for harvesting after the end-effector was pre-positioned on the apple's stem. However, the picking time reported by Sakai, Iida, Osuka \& Umeda (2008) included the time required for the manipulator and end-effector to reach, pick, and deposit the fruit. In some other articles, it could not be determined whether reported times reflected the picking time or the total cycle time. If we used the data presented about the machine vision results and cycle time to assume about the picking time, a question mark was placed beside the table entry. A significant number of references (41\%, or 16 out of 39$)$ did not report any results related to picking time. The lowest reported picking time was 1 second (Scarfe, Flemmer, Bakker \& Flemmer, 2009), and the average picking time of the 23 reported was approximately 11.5 seconds. There was also significant variation in sample sizes and testing conditions (i.e., laboratory conditions versus field trials).

\subsection{Formal Design Process}

Nine of the 39 technologies, or $23 \%$, used formal design processes during manipulator and end-effector development. All the technologies provided at least a qualitative analysis of the environment and functional requirements. It is possible that the remaining 30 technologies did incorporate some types of design methodology; however, the application of such methods was not readily apparent in the project publication.

\subsection{Manipulator DOF and Actuation}

Of the 39 technologies reviewed, three did not report the type of actuators used in the manipulator (and we were unable to determine the type of actuators from the pictures included in the publication). Seven of the 39 technologies (or 17\%) incorporated commercially available robotic manipulators with electric actuators. The breakdown of the manipulator actuators used in the remaining 29 technologies that developed their own custom manipulators is shown in Figure 3.

Many of the technologies did not identify the reasoning for the selection of the chosen manipulator actuators. Most of the technologies (20/29) that developed their own manipulators used only electric actuators. For harvesting operations where the weight of the manipulator structure and agricultural payload is expected to be low, electric servomotors and stepper motors are often advantageous because they are relatively easy to control and provide fast response times. Another significant advantage of electric motors for agricultural operations is that they do not pollute the working environment. Only $17 \%(5 / 27)$ of the technologies reported using pneumatic and hydraulic actuators in their manipulator designs. Because of their higher power-to-weight ratios, pneumatics and hydraulics were adopted for larger structures with higher payloads. For example, Foglia \& Reina (2006) used pneumatics to support a $16 \mathrm{~kg}$ end-effector. Compared to hydraulics, pneumatic actuators are faster but more difficult to control.
Depending on the working environment, a potential disadvantage of hydraulics is oil leakage from the actuators. Both hydraulic and pneumatic actuators require more complex power systems than electric motors, which could reduce overall system mobility. The results of the review did not indicate a direct correlation between the actuation scheme and manipulation performance as measured with picking time. Therefore, the selection of the manipulator actuators depends on the design criteria for static/dynamic loads, accuracy, speed, mobility, and cost. Also, only three of the technologies reported the integration of on-line collision avoidance methods. For two of these technologies (Tanigaki, Fujiura, Akase \& Imagawa, 2008; Van Henten et al., 2002), the working environment was a greenhouse with vertical distribution of the crop.

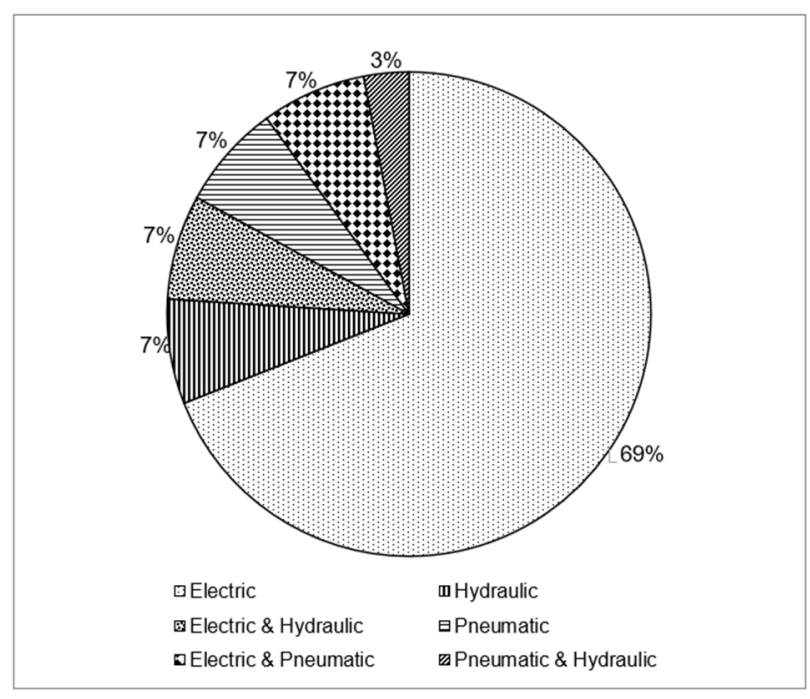

Figure 3. Breakdown of manipulator actuators used in reviewed technologies.

\subsection{Fruit Contact Locations and Methods of Detachment}

In order to detach the crop from the plant, the end-effector must contact the fruit or its stem. The majority (44\%) of the end-effectors contacted both the fruit and its stem. Less than a quarter $(23 \%)$ of the end-effectors contacted only the stem and $33 \%$ employed fruit as the contact for detachment; cutting the stem was considered stem contact.

The type of detachment methods used included gripping with suction and/or grippers, grasping, and stem cutting. The most common method $(16 / 39$ or $41 \%)$ of detachment was the combination of gripping and cutting. Only one of the end-effectors produced a grasp adaptive to the shape of the fruit. The breakdown of the project detachment methods is provided in Figure 4. None of the end-effectors employed dexterous manipulation of the crop during the detachment process. All technologies that used gripping only (14/39) during fruit picking used end-effector forces applied by the manipulator to assist with the detachment process. This suggests that for those techniques that apply gripping only, end-effector forces required for fruit detachment should be a performance specification for the adopted manipulator. 


\subsection{End-Effector Actuation and Sensing}

The number of actuators used in the end-effector prototypes varied from one to a maximum of four. Most of the technologies used one or two actuators in their end-effector designs (Fig. 5). Most of the actuators were either electric or pneumatic - only one hydraulic actuator was used in the fabrication of an end-effector (Fig. 6). Also, it is important to highlight that the source for the compressed gas (tanks, compressors, etc.) was not located on the end-effector.

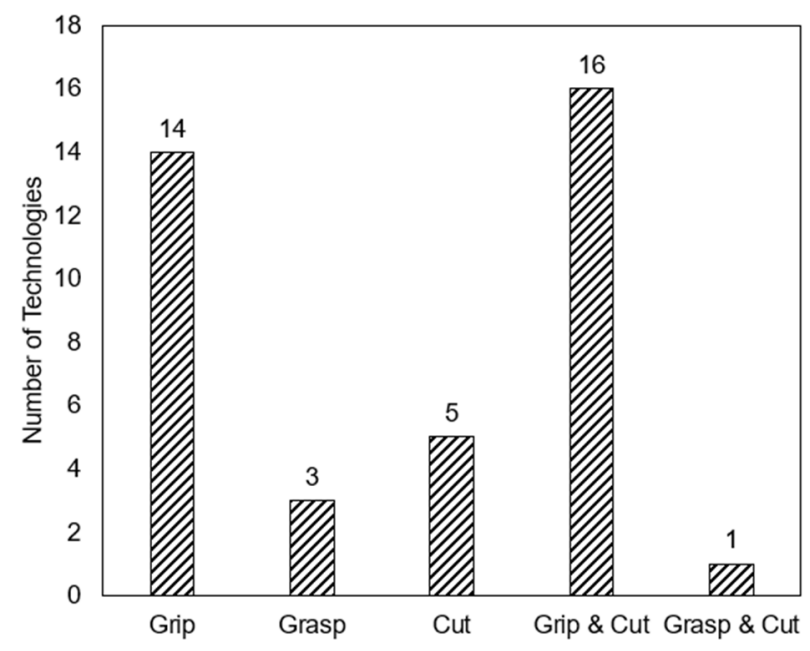

Figure 4. Detachment methods employed to remove the fruit from the plant.

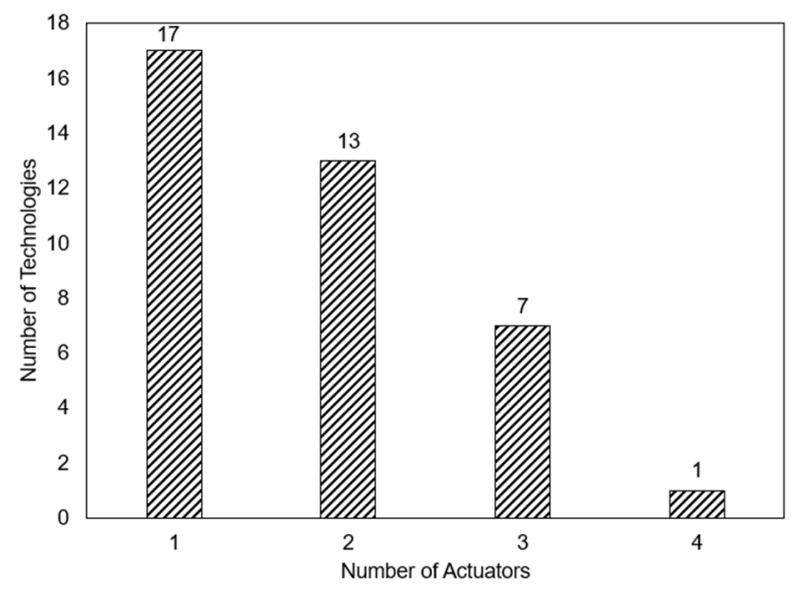

Figure 5. Number of end-effector actuators used in reviewed technologies.

Approximately half of the technologies (17/39) reported the installation of hardware designed to provide sensory feedback during the end-effector's interaction with the environment. For those technologies that incorporated end-effector sensors, the number used varied from one to eight (Table 1). It was expected that many of the technologies would include force or pressure sensors to control gripping force and minimize bruising. However, because many of the end-effectors grasped the stem only and/or used suction, few of the technologies incorporated force sensors (3/39).
About 50\% (21/39) of the reviewed studies reported success rate in detaching fruit. Roughly $75 \%(15 / 21)$ of those studies achieved a detachment rate of $75 \%$ or more. Of those 15 studies, 14 used electrical actuations in their manipulator design. The manipulators used have varying degrees of freedom (DOF; 3-7). Most of the studies with lower than $75 \%$ detachment rate have generally smaller DOF $(<5)$. On average, however, robotic systems with $6 \mathrm{DOF}$ or less achieved a $77 \%$ success rate whereas the systems with 6 or higher DOF achieved around $82 \%$ average success rate. Most of the studies (12 of 15 technologies) with detachment success rate $>75 \%$ used a gripping mechanism for fruit detachment. The choice of end-effector design for the technologies with success rate $>75 \%$ utilized either pneumatic or electric actuation. These findings show that robotic systems for fruit picking might benefit from using electrical actuation for manipulators and electrical and pneumatic actuation for end-effectors with gripping mechanism for grabbing and detaching fruit. It was also found that a manipulator with 6 or more DOF might be desirable to achieve high detachment efficiency in fruit and vegetable picking.

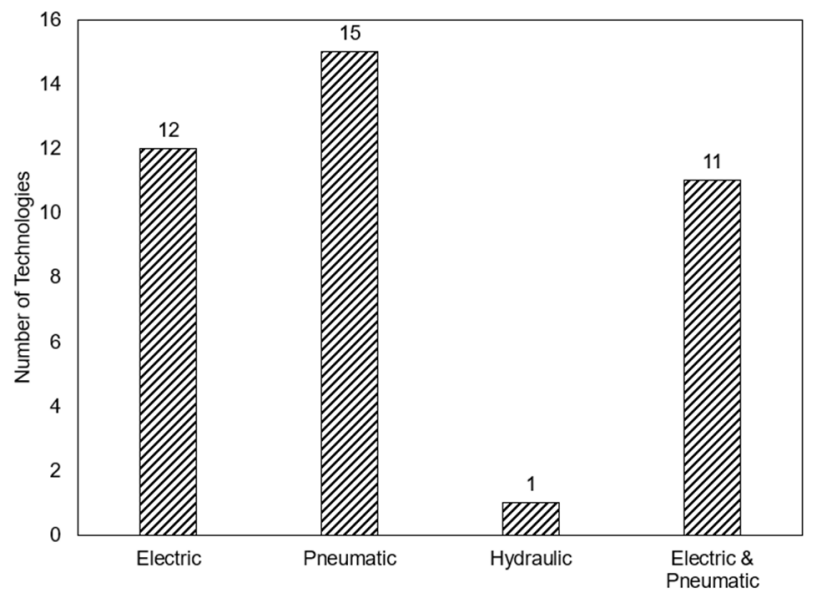

Figure 6. Type of end-effector actuators used in reviewed technologies.

\section{Discussion}

Despite extensive research and development over the past several decades, we do not know of any successful commercial implementations of robotic harvesting systems for specialty crops. In addition to overall harvest success and reduced damage rates, a good indicator of system performance is the rate of robotic harvesting compared to manual harvesting. A comparison of robotic picking times of several crops compared with the reported rates for skilled, commercial pickers is shown in Figure 7. The data indicate that manual picking rates are significantly better than the current rates of robotic picking. Admittedly, the sample size is small, as the chart presents the average picking speed from a sample size of only one to three technologies. Note that the reported picking time, which does not include set-up times or the time required for machine vision processing, compares the speed of robotic manipulation (i.e., reach, picking, and fruit storage) with the equivalent human activities as a measure of manipulation performance. Not considering other factors that could affect commercial implementation, current data indicates that there is significant opportunity for improvement in picking times. 


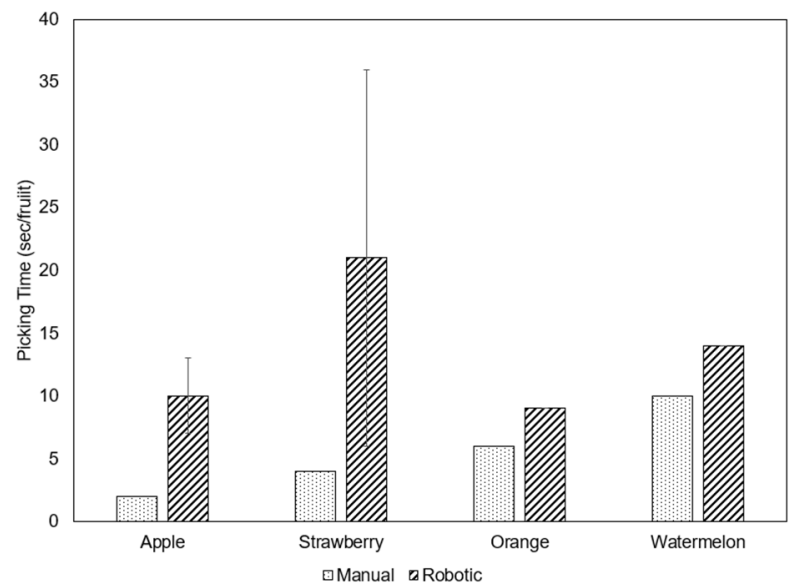

Figure 7. Comparison of robotic picking time with manual picking rates.

The results of our review indicate that advances in manipulation performance during specialty crop harvesting have been limited by the following four factors:

1. Absence of standard reporting conditions and performance indicators makes it difficult to compare techniques and identify best practices, 2 . Functional requirements and performance specifications were insufficiently defined, and manipulators and end-effectors were not optimized for the workspace and task, 3. End-effectors were insufficiently robust to errors in position input provided by vision systems; and 4 . Systems were highly reliant on sensory feedback with insufficient use of model-based control supported by characterization of fruit and plant properties

The problem of insufficiently defined functional requirements and performance indicators as applied to the entire robotic system has already been thoroughly described by Bac et al. (2014). While the development of quantitative performance measures is beyond the scope of this review, we believe that the field must adopt standardized reporting parameters to aid benchmarking. Some possible criteria for measuring manipulation performance include:

- Picking time (sec) - the average time required to harvest a single fruit in the system's reachable workspace. This measurement begins when position data is provided to the manipulator (i.e., fruit localization is completed) and ends when the detached product is stored. Therefore, it includes the computation time required for inverse kinematics and motion planning calculations.

- Detachment success (\%) - the fruit successfully detached and stored as a percentage of total number of fruits the system attempts to pick.

- Damage rate (\%) - total damage occurring to the fruit in the system's workspace as a percentage of all fruit present. This includes any damage to fruit that would preclude its distribution on the fresh market as well as any damage to the plant that would prevent it from producing additional fruit. Damage to fruit that the system does not attempt to pick should also be included.

Unfortunately, because most agro-robotics research has focused on machine vision, mechanical design optimization procedures for harvesting manipulators are not well documented in the literature. The earliest tree fruit-picking robots typically incorporated articulated or spherical manipulators with 3-4 degrees of freedom (Grand D'Esnon, Rabatel, Pellenc, Journeau \& Aldon, 1987; Harrell \& Levi, 1988). The harvesting efficiency for the early prototype systems was approximately $75 \%$. This relatively low performance level was attributed to poor fruit identification and the inability to negotiate natural obstacles inside the tree canopy (Sarig, 1993) due to the manipulator's limited degrees of freedom (DOF). It is important to note that researchers in the 1980s deliberately designed manipulators with less coupling between the degrees of freedom so that the control system of the mechanical components could be simplified thereby enabling dedication of most processor capacity to the vision system (Muscato, Prestifilippo, Abbate \& Rizzuto, 2005). Because of increased computational power, the time required for today's processors to numerically determine feasible solutions to the inverse kinematics problem is not usually significant. For systems operating in complex environments that require motion planning and obstacle avoidance, like the cucumberpicking robot (Van Henten et al., 2003), motion planning computations required a significant portion of execution time. Furthermore, effective motion planning can also optimize trajectory costs including power requirement, operational time, and uncertainty. Recent developments in algorithms as well as increased computing capacity should also help minimize computation time for the motion planning subtask. However, it is important to realize that in the unstructured harvesting environment that requires obstacle avoidance, the complexity of motion planning will significantly increase with the DOF of the robot system.

Most technologies reviewed incorporated kinematic designs based on qualitative assessments of the workspace. For technologies that provided specific guidelines used during design of the manipulator, the criteria typically included a workspace compatible with the structure of the plant or simplified control requirements. Song, Sun, Zhang, Zhang \& Xu (2007) reported a design procedure used to optimize two parameters, the length of the upper arm and forearm, of a manipulator with four rotational links in order to obtain the most compact mechanical structure compatible with the growth pattern and fruit distribution range of greenhouse eggplants. The use of software simulations to analyze manipulator workspace compatibility has also been described by Fengying, Guolong, Xiangjun, Zhen \& Ce (2011), Li, Liu, Li \& Li (2008), Shamshiri et al. (2018c), Sun, Zou, Zou, Chen \& Cai (2010), and Wang et. al. (2018). While these references are not complete technologies presented in Table 1, they describe methods relevant to manipulator design and thus are worth highlighting. Other researchers (Arima, Kondo, Yagi, Monta \& Yoshida, 2001; Arima, Monta, Namba, Yoshida \& Kondo, 2003; Foglia \& Reina, 2006; Zhao, Lu, Ji, Zhang \& Chen, 2011) have made their primary goal the creation of mechanical designs with quasi-linear behavior and, hence, relatively simple control requirements.

The use of system design processes and quantitative performance measures to optimize manipulator parameters was described by three technologies and represents a best practice. Edan, Haghighi, Stroshine \& Cardenas-Weber (1991) and Edan \& Miles (1993, 1994) used extensive system design procedures in the development of a mechanical manipulation system for a melon harvesting robot. Van Henten, Van't Slot, Hol\& Van Willigenburg (2009) presented a detailed procedure that used quantitative performance measures to optimize the kinematic structure of an autonomous robot for harvesting greenhouse cucumbers. More specifically, the quantitative performance criterion combined measurements for both collision-free path length and manipulator dexterity, and the optimization problem was solved by using the "DIviding RECTangles" (DIRECT) algorithm (Jones, Perttunen \& Stuckman, 1993) implemented in the TOMLAB package (TOMLAB Optimization, Uppsala, Sweden). Based on 
the results of this computationally intensive analysis, a four link PPRR (2 Prismatic 2 Revolute) manipulator was found to be enough for picking greenhouse cucumbers. Highlighting the utility of this optimization study, they found that the less expensive, simpler 4-DOF design could be used in place of the original 6-DOF, Mitsubishi RV-E2 (Mitsubishi Electric Automation, Inc., Vernon Hills, IL) adopted for their original field test of an autonomous cucumber harvesting robot (Van Henten et al., 2003). Another interesting finding was that their optimization results indicated that Cartesian manipulators, which are frequently used in agricultural harvesting research (Edan, Rogozin, Flash \& Miles, 2000; Reed, Miles, Butler, Baldwin \& Noble, 2001), performed poorly for the chosen design criteria compared to other kinematic structures.

The third technology that utilized quantitative performance measures during manipulator design was the watermelon harvester developed by Sakai, Iida, Osuka \& Umeda (2008). This project defined a novel, global performance index for a mobile manipulator. Also, the specification of design criteria and subsequent use of performance indices to select a kinematic structure was particularly noteworthy. Manipulability ellipsoids (Yoshikawa, 1985) were used to assess the normalized endpoint force in the vertical direction, and the normalized work space volume (Yang \& Lee, 1984) was used to compare the normalized workspaces of parallel, Selective Compliance Assembly Robot Arm (SCARA) type, cylindrical, and Cartesian manipulators. It is interesting to note the role that the plant environment had influenced the design objectives stated by Van Henten et al. (2009), who wanted to optimize dexterity, and Sakai et al. (2008), who emphasized vertical endpoint force at the expense of manipulability. For the relatively unstructured cucumber growing environment, dexterity is more important criteria. Whereas for the heavier watermelon harvested at ground level, endpoint force was valued over manipulability.

Based on the results of the review, manipulation methods incorporating single DOF gripping was the most prevalent approach. Gripping the stem only was sometimes viewed as advantageous because by minimizing contact with the fruit, the likelihood of damage was reduced. Unfortunately, for systems where the robot only made contact with the stem, damage rates to the fruit or plant were not reported. For tree fruit especially, there seems to be a direct relationship between stem contact and fruit damage. The primary source of damage for systems making only fruit contact (Baeten, Donne, Boedrij, Beckers \& Claesen, 2008; Grand D'Esnon, 1985; Pool \& Harrell, 1991) was either a stem-pull or spur detachment. Bruising or puncturing of the fruit was not described. Gripping of the fruit and/or stem requires precise position data. However, the stem can be particularly difficult for vision systems to detect and identify because of its small size and tendency to be occluded. Some degree of position error in the fruit and stem coordinates is to be expected. In general, gripping methods are less robust to position error and variable fruit shapes. Feng, Zheng, Qiu, Jiang $\&$ Guo (2012) stated that position errors of just $6 \mathrm{~mm}$ would cause a picking failure. Hayashi et al. (2010) noted that 3D positional errors and peduncle detection accounted for their system's picking failures. Also, Van Henten et al. (2003) stated that inaccurate positioning of the end-effector due to error accumulated during visual localization was the largest source of picking failures.

It was apparent that each of the technologies proceeded with a qualitative understanding of the preferred method for manual harvesting of the fruit under consideration. However, only $25 \%$ of the technologies (9/36) specifically mentioned conducting either their own studies or referencing already completed studies about the physical properties of the fruit and forces required during harvesting. The characterization of properties like stem tensile strength and fruit bruising thresholds may not be critical for all fruit removal methods, for example the use of a stem cutter. Still, an endeffector design process facilitated by thorough knowledge of these properties represents a good practice because of the increased likelihood for successful fruit detachment with minimal damage. As an example, by extensively studying bruising and the torque required for mushroom detachment, Reed et al. (2001) were able to design an end-effector that produced lower damage rates than those occurring during manual harvesting.

In the past fifteen years, researchers and engineers have incorporated many different creative fruit removal techniques in end-effector designs. Because of our own interest in the development of an apple harvesting robot, we have attempted to identify and compare the advantages and disadvantages of the techniques used for apple harvesting (Table 2). These considerations may still be applicable to different harvesting environments and fruit.

Table 2. Advantages and Disadvantages of Different Fruit Removal Techniques in the Context of Apple Harvesting.

\begin{tabular}{|c|c|c|}
\hline $\begin{array}{c}\text { Fruit Removal } \\
\text { Method }\end{array}$ & Pros & Cons \\
\hline $\begin{array}{l}\text { Gripping of } \\
\text { the peduncle }\end{array}$ & $\begin{array}{l}\text { Minimizes contact with the } \\
\text { fruit, lessening the } \\
\text { likelihood of damage } \\
\text { Small forces are required } \\
\text { to remove the fruit by } \\
\text { lifting and twisting } \\
\text { Reduces the likelihood of } \\
\text { stem pulls }\end{array}$ & $\begin{array}{l}\text { Requires precise location of the } \\
\text { peduncle, which can be difficult } \\
\text { to detect against } \\
\text { leaves/branches and in fruit } \\
\text { clusters } \\
\text { The path/approach to the fruit is } \\
\text { constrained }\end{array}$ \\
\hline $\begin{array}{l}\text { Vacuum } \\
\text { Suction }\end{array}$ & $\begin{array}{l}\text { Lessens the likelihood of } \\
\text { fruit damage } \\
\text { Fewer actuators and } \\
\text { sensors are required } \\
\text { Greater flexibility for path } \\
\text { planning }\end{array}$ & $\begin{array}{l}\text { Adjacent fruit and branches can } \\
\text { interfere with efficient } \\
\text { operation } \\
\text { Higher incidence of stem pulls }\end{array}$ \\
\hline $\begin{array}{l}\text { Cutting } \\
\text { (thermal or } \\
\text { mechanical) }\end{array}$ & $\begin{array}{l}\text { Possibly a faster removal } \\
\text { method } \\
\text { Minimizes the likelihood } \\
\text { of stem pulls } \\
\text { Thermal cutting can reduce } \\
\text { the likelihood of disease } \\
\text { transfer }\end{array}$ & $\begin{array}{l}\text { More complex control } \\
\text { requirements, which can lead to } \\
\text { higher computational \& } \\
\text { economic costs } \\
\text { May require precise location of } \\
\text { fruit peduncle }\end{array}$ \\
\hline
\end{tabular}

\section{Possible Lines of Future Research}

To address some of the previously discussed challenges limiting the effectiveness of robotic harvesting systems, we now propose potential lines of future research that may help realize positive trends in system performance. Our recommendations focus on manipulator optimization, enhanced end-effector robustness, alternative sensing techniques, alternative manipulation technologies, and human-machine collaboration.

\subsection{Manipulator Optimization}

Looking to the future, systematic approaches for agricultural automation that combine machine design and horticultural practices remain an opportunity for improvement. As discussed by both Sanders (2005) and Peterson (2005), it is widely recognized that to maximize the potential of 
robotic fruit harvesting, compatible horticultural systems are required. In these systems, plant characteristics, like canopy growth, tree spacing, and fruit position, are developed in conjunction with machine designs. Historically, growers were unwilling to totally transform orchards and fields to accommodate robotic harvesters because of the risks and expenses involved in making a change for an unproven technology (Muscato et al., 2005). Specific recommendations include evenly distributed fruit located away from main obstacles like trellis wires, thick branches, and trunks. Based on the results of this review, fruit clustering appears especially problematic because adjacent fruits tend to interfere with the mechanical detachment method. Also, even fruit distribution in planar arrangements can potentially reduce the DOF required and simplify motion planning requirements, which could lead to improvements in picking times. In Washington State, most of the new apple acreage is being planted to a twodimensional, planar canopy supported by a wire and post trellis system whereby most of the branches and fruit are visible and accessible to machines. This trend is in accordance with the general goal of improving productivity using simple, narrow, accessible, and productive (SNAP) canopies. The authors expect that this trend of planting and training trees to an architecture with more accessible fruit will simplify manipulator optimization and help improve system performance. Yet another important horticultural consideration that should be studied is the importance of stem attachment. As mentioned earlier, in the fresh market apple industry stem pulls are considered undesirable because some studies have shown that they may predispose certain apple cultivars to disease (Janisiewicz \& Peterson, 2004). Still, the importance of stem attachment is a source of some debate within the industry. More studies are needed to conclusively determine whether a stem pull that does not cause fruit damage increases the likelihood of postharvest disease. If additional testing shows that stem attachment is not critical for market acceptance, a significant constraint of mechanical harvesting will be removed.

In order to optimize the manipulator for the required task, future research technologies should first characterize the workspace using the crop row spacing (if applicable), plant spacing, crop height, fruit distribution within the plant, and patterns of fruit orientation. The application of formal design processes that first define functional requirements and then develop detailed performance specifications for kinematic requirements, manipulator payload, motion planning requirements, end-effector forces, etc. are recommended. As discussed by Zhang, Yao \& Du (2014), there have been many manipulator kinematic performance measures proposed in the literature, several of which are directly applicable to harvesting applications. Selecting the correct performance indices can be a confusing task. The collision-free path length and manipulator dexterity (Baur, Pfaff, Ulbrich \& Villgrattner, 2012) at the fruit location are particularly relevant. The collision-free path length, which was studied by Van Henten et al. (2009), is a useful optimization parameter for all agricultural harvesting applications because of its influence in determining manipulator speed. Lehnert, Perez \& McCool (2015) have also done some preliminary work on the optimization of a manipulator to minimize the collision-free path and maximize dexterity. Though the importance of manipulator dexterity, or manipulability, at the location of the fruit is application-dependent, use of these indices during manipulator optimization should be a goal.

Other important considerations for future manipulator research include multi-tasking, modular configurations, and fault tolerance. Because of the expenses involved in the development and procurement of robotic systems, a modular design with multiple configurations that can be continuously used throughout the year for different tasks like pruning, thinning, pesticide application, and harvesting is ideal. Research on the development of a multi-purpose prototype for greenhouse applications was recently reported (Belforte, Deboli, Gay, Piccarolo \& Aimonino, 2006). A modular configuration where an agricultural technician could quickly and easily replace a defective component is also advantageous. Agricultural technicians may not have extensive training on robotic hardware, so a design that permits relatively simple repairs is desirable. Harvesting windows are short and equipment downtime can have significant economic consequences. Another option for consideration is fault tolerance, which is the property of a system to continue to function in the event of a failure or internal fault.

Recently, commercial robotic manipulators such as Sawyer and UR5 from Universal Robots are gaining high attention in agricultural operations such as harvesting (Soria et al., 2018; Sukkar, 2018). These industrial robotic manipulators will provide better path planning and trajectory optimizations in high-dimensional configuration space. The arm with six DOF allows operating the robot in any arbitrary position in 3D space (Hayashi, 1994; Soria et al., 2018; Sukkar, 2018), making them suitable for different agricultural operations, including harvesting. These commercial manipulator arms can be configured using Robot Operating System (ROS) environment which provides flexibility in many different aspects such as simulation and obstacle avoidance using openRAVE (Diankov, 2019; OpenRAVE, 2013; Shamshiri, Hameed, Karkee \& Weltzien, 2018d) motion planning using TrajOpt (Koval \& Velgapudi, 2016; Schulman et al., 2013).

\subsection{Enhanced End-Effector Robustness and Sensing}

The transfer of industrial robotic technology directly to field-based, biologically driven environments has resulted in limited success at mechanizing specialty crop agriculture. A general desire to replicate the motions used during manual harvesting of fruit has helped guide the development of agricultural end-effectors, which is a reasonable objective considering that the techniques used by professional pickers have been adopted because of their efficiency. However, design criteria have not been sufficiently defined because of a lack of fundamental knowledge about the dynamics of hand-picking operations. Future end-effector designs should be facilitated by thorough analysis of hand and finger motions and the force/torque necessary to detach the fruit with the stem still attached. Study of the forces and torques involved in the process and the trajectory of hand and fruit movement are critical pieces of information. An example of a study designed to facilitate an effective apple-picking end-effector is the recent experimental analysis by Tong, Zhang, Karkee, Jiang \& Zhou (2014). This study determined the forces required for different picking patterns and stem orientations. Though not the subject of this review, additional comprehensive studies of the natural growth habits of fruits and trees, like stem length, stem stiffness, and fruit orientation, are also crucial to obtain a better understanding of how to detach fruits effectively and efficiently.

To date, most end-effector designs have incorporated gripping techniques with limited degrees of freedom. Another opportunity for future end-effector research is the incorporation of underactuated devices. Underactuated designs have become a very popular area of research in the robotic grasping community and offer several advantages for agricultural harvesting applications. A review of underactuated robotic hands has been completed by Birglen, Laliberte \& Gosselin (2008). Simply defined, underactuated hands have fewer actuators than degrees of freedom and provide a shape-adaptive grasp that bridges the gap between complex, fully actuated robotic hands and simple grippers. Dollar \& Howe (2010) showed 
that their Shape Deposition Manufacturing (SDM) Hand can passively adapt to the shape of a target object even in the presence of largepositioning errors. During harvesting, fruit picking patterns are consistent and multiple grasp postures are typically not required. Likewise, none of the reviewed technologies incorporated in-device manipulation of the fruit. Therefore, because dexterous grasping is unnecessary, under-actuation may be particularly suitable for harvesting end-effectors. Devices that can passively adapt to variable fruit shape, size, and orientation even in the presence of positioning errors, which can be expected from machine vision input, are highly desirable for picking applications and may help realize improvements in robustness. Underactuated mechanisms could also be used for devices that only contact the fruit stem.

We also believe that another direction of research that could provide performance improvements is the study of "under sensed" systems. In these systems few or no sensors would be used to provide feedback about the end-effector's interaction with the target fruit. For example, consider a system that integrates a global camera with an end-effector designed to provide robustness bounding the maximum expected input position error. Such a system would not require visual servoing or other types of sensors for final fruit localization. With an accurate environmental model developed through detailed analysis of the forces required to detach the fruit, optimal detachment force patterns could be produced using modelbased, feedforward control. Interaction forces between the end-effector and fruit could be controlled by actuation force with minimal or no use of sensors for feedback. The SDM Hand (Dollar \& Howe, 2010) demonstrated robust grasping utilizing open-loop, feedforward control. Systems combining position robustness and model-based control with minimal use of sensors could potentially improve performance and lower costs and design complexity.

There are also opportunities for improvements in end-effector manufacturing processes. Two of the picking end-effectors were fabricated with additive manufacturing techniques. This is not surprising considering that additive manufacturing technologies have only recently become widely adopted in manufacturing processes. Using rapid prototyping for endeffector components can significantly reduce design complexity and cost. The weight of end-effectors produced with 3D printers can also be much less compared to previous designs incorporating standard metal components. The use of flexure joints in end-effector designs rather than traditional revolute joints with bearing and springs presents additional advantages. Passively compliant joints like those used in the SDM hand (Dollar \& Howe, 2010) reduce joint oscillations and deflect out of plane during unintended collisions - like those that can be expected in the unstructured crop environment - thereby improving durability. An example of an underactuated end-effector produced with additive manufacturing is the device recently developed by the authors and shown in Figure 8 .

\subsection{Alternative Manipulation Technologies}

The standard approach to robotic harvesting has involved the integration of a vision system, serial link manipulator, and picking end-effector to selectively harvest individual ripe fruit. Other options for future study include the use of parallel-link robots, like the Delta robot, for agricultural harvesting. For light payloads and fast cycle times in small workspaces, the delta type robot often exceeds the performance of serial link robots. Additional DOF and actuation may be needed to place a delta configuration near the crop. The following conceptual ideas proposed require a fundamental shift in the approach to robotic harvesting of specialty crops.

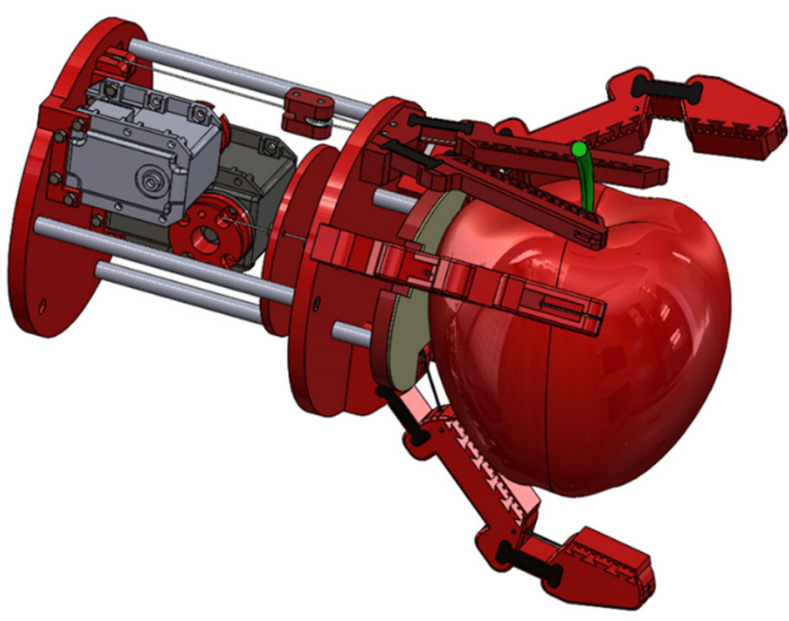

Figure 8. Underactuated end-effector used for robotic apple harvesting. Reproduced with permission from the authors (Davidson \& Mo, 2015).

In the introduction of this review we mentioned that researchers have attempted to use shake-and-catch systems that applied vibratory motion to the entire plant. This approach towards mass harvesting of fruit produced results that were unacceptable for fresh market crops. In trellis apple or other fruit orchards, detaching and catching the fruit by shaking small localized regions of the tree with robotic end-effectors could be a direction for future investigation. Energid Technologies (Cambridge, Massachusetts) is taking a different approach and has recently undertaken efforts to develop a citrus fruit picker that combines selective harvesting with mass removal techniques (Aloiso, Mishra, Chang \& English, 2012). Their novel concept uses a so-called "Frog Tongue" mechanism consisting of a flexible tube powered by compressed air that rapidly extends/retracts from its base and severs the stem of the fruit. Multiple machine vision cameras provide input to tracking algorithms that direct and guide the tubes during extension through control of a 2-DOF linear positioning unit. The company eventually intends to mount multiple grids of 64 or more such picking mechanisms. It is not known whether a catch system will be used or if the fruit will go to the fresh market or process market. The reported picking speed was 2-3 seconds per orange.

Other robotics technologies that could be incorporated in harvesting systems include soft robotics, an area of much recent research (Rus \& Tolley, 2015), and autonomous micro air vehicles. Soft robotics is a rapidly developing field that is replacing hard robotic systems with soft robots composed entirely or primarily of low-modulus or flexible-inextensible materials actuated by pneumatics (Wehner et al., 2014). Because of their compliance, devices incorporating the technology can provide robust, adaptive grasps in unstructured environments (Deimel \& Brock, 2014). The materials are also inexpensive, lightweight, robust to blunt impact, and minimize damage during unplanned collisions. These are benefits particularly advantageous for harvesting end-effectors that are used in unstructured environments to manipulate delicate products. Also, micro air vehicles (MAVs) are now used in many civil applications. Recently, MAVs that can perform autonomous vision-based navigation have been proposed (Schmid, Lutz, Tomic, Mair \& Hirschmuller, 2014). We suggest that a fleet of harvesting MAVs with vision and picking systems could be released into a field or orchard for autonomous harvesting. Admittedly, realization of this concept would require significant research and development. 


\subsection{Human-Machine Collaboration}

The final research direction that we suggest for improved harvesting performance is human-machine collaboration and haptic interfaces. Technologies evaluated in this paper focused on completely autonomous systems with no user input. A semi-autonomous system that integrated human-machine collaboration could potentially improve overall harvest success rates. For example, if the machine vision system was unable to identify the fruit because of occlusion or clustering, the system operator could use a haptic interface and camera feed to manually complete the picking sequence with the robot replicating operator's physical motions. This type of interface would eliminate the need for manual harvesting of unpicked fruit in the robot's reachable workspace.

\section{Summary}

This review examined the current status of manipulator and end-effector technologies investigated for various types of fruit and vegetable harvesting with a focus on mechanical design and manipulation performance. Because of its interdisciplinary nature, dissemination of research results often occurs across multiple publication platforms. We have consolidated the results of 39 different harvesting technologies completed since 1986 to identify trends and issues. Challenges to commercial adoption related to manipulation performance have been discussed and best practices were noted. We have also presented some potential avenues of future research in manipulation and end-effector technologies that may help improve overall system performance. While our specific scope of review was the mechanical design of the manipulation system, it is also noted that a systematic and integrated approach utilizing expertise from disciplines such as horticulture, computer science, engineering, and economic and social sciences is needed for the successful development and commercial adoption of automated harvesting systems for specialty crop production. Even though, the robotic systems researched and developed so far cannot compete to human workers in terms of harvesting speed and efficiency, actively ongoing research efforts around the world coupled with strong funding commitments from government and private organizations show potential for practically adaptable solutions in the near future. Some of the key findings of this study are as follows:

1. It is important that future research and development on robotic systems consider manipulator optimization, enhanced end-effector robustness, workspace optimization, alternative sensing techniques, alternative manipulation technologies, and human-machine collaboration.

2. Robotic systems for fruit picking might benefit from using electrical actuation for manipulators and electrical and pneumatic actuation for end-effectors with a gripping mechanism for grabbing and detaching fruits.

3. Commercial robotic manipulators (such as UR5 from Universal Robots) provide better path planning and trajectory optimizations in high-dimensional space, which might be exploited in the future for prototype development and concept evaluation.

4. Robotic manipulators with six DOF allow operating the robot in any arbitrary position in $3 \mathrm{D}$ space, making them suitable for not only harvesting but also other agricultural operations such as pruning and thinning.

5. Virtual environments and simulation software can be a potential tool for accelerating research and development in agricultural robots including end-effectors and the manipulator design and prototyping.
These tools not only provide year-round opportunities for evaluating the agricultural robots but also help in enhancing system performance (increasing efficiency, accuracy, speed, and minimizing fruit damage).

\section{Acknowledgments}

This research was supported in part by the United States Department of Agriculture (USDA)'s Hatch and Multistate Project Funds (Accession No 1005756 and 1001246), USDA National Institute for Food and Agriculture competitive grant (Accession No 1000339), and Washington State University (WSU) Agricultural Research Center (ARC). Any opinions, findings, and conclusions expressed in this publication are those of the authors and do not necessarily reflect the view of the USDA or Washington State University.

\section{References}

Aljanobi, A., Al-hamed, S., \& Al-Suhaibani, S. (2010). A Setup of Mobile Robotic Unit for Fruit Harvesting. $19^{\text {th }}$ International Workshop on Robotics in Alpe-Adria-Danube Region, (pp. 105-108). Budapest, Hungary.

Aloiso, C., Mishra, R. K., Chang, C., \& English, J. (2012). Next Generation Image Guided Citrus Fruit Picker. IEEE Conference on Technologies for Practical Robot Applications (TePRA), (pp. 37-41). Woburn, MA, USA.

Antonelli, M., Auriti, L., Beomonte Zobel, P., \& Raparelli, T. (2011). Development of New Harvesting Module for Saffron Flower Detachment The Romanian Review Precision Mechanics, Optics \& Mechatronics, 39, 163-168.

Arima, S., Kondo, N., Yagi, Y., Monta, M., \& Yoshida, Y. (2001). Harvesting Robot for Strawberry Grown on Table Top Culture (Part 1), Harvesting Robot Using 5 DOF Manipulator. Journal of Society of High Technology in Agriculture, 13(3), 159-166.

Arima, S., Monta, M., Namba, K., Yoshida, Y., \& Kondo, N. (2003). Harvesting Robot for Strawberry Grown on Table Top Culture (Part 2), Harvesting Robot with a Suspended Manipulator under Cultivation Bed. Journal of Society of High Technology in Agriculture, 15(3), 162-168.

Bac, C. W., van Henten, E. J., Hemming, J., \& Edan, Y. (2014). Harvesting Robots for High-value Crops: State-of-the-art Review and Challenges Ahead. Journal of Field Robotics, 31(6), 888-911.

Baeten, J., Donne, K., Boedrij, S., Beckers, W., \& Claesen, E. (2008). Autonomous Fruit Picking Machine: A Robotic Apple Harvester. Field and Service Robotics, 42, 531-539.

Baur, J., Pfaff, J., Ulbrich, H., \& Villgrattner, T. (2012). Design and Development of a Redundant Modular Multipurpose Agricultural Manipulator. IEEE/ASME Inernational Conference on Advanced Intelligent Mechatronics, (pp. 823-830). Kaohsiung, Taiwan.

Belforte, G., Deboli, R., Gay, P., Piccarolo, P., \& Ricauda Aimonino, D. (2006). Robot Design and Testing for Greenhouse Applications. Biosystems Engineering, 95(3), 309-321.

Birglen, L., Laliberte, T., \& Gosselin, C. (2008). Underactuated Robotic Hands, Springer Tracts in Advanced Robotics (Vol. 40). Springer.

Bulanon, D. M., \& Kataoka, T. (2010). Fruit Detection System and an End Effector for Robotic Harvesting of Fuji Apples. Agricultural Engineering International: CIGR Journal, 12(1), 203-210.

Burks, T., Villegas, F., Hannan, M., Flood, S., Sivaraman, B., \& Subramanian, V. (2005). Engineering and Horticultural Aspects of Robotic Fruit Harvesting: Opportunities and Constraints. HortTechnology, 15(1), 79-86.

Cardenas-Weber, M., Stroshine, R., Haghighi, K., \& Edan, Y. (1991, MayJune). Melon Material Properties and Finite Element Analysis of Melon 
Compression with Application to Robot Gripping. Transactions of the ASAE, 34(3), 920-929.

Chatzimichali, A. P., Georgilas, I. P., \& Tourassis, V. D. (2009). Design of an Advanced Prototype Robot for White Asparagus Harvesting. IEEE/ASME International Conference on Advanced Intelligent Mechatronics, (pp. 887892). Singapore.

Cho, S., Chang, S., Kim, Y., \& An, K. (2002). Development of a Threedegrees-of-freedom Robot for Harvesting Lettuce using Machine Vision and Fuzzy Logic Control. Biosystems Engineering, 82(2), 143-149.

Cutkosky, M. R. (1989). On Grasp Choice, Grasp Models, and the Design of Hands for Manufacturing Tasks. IEEE Transactions on Robotics and Automation, 5(3), 269-279.

Davidson, J. R., \& Mo, C. (2015). Mechanical Design and Initial Performance Testing of an Apple-Picking End-Effector. ASME International Mechanical Engineering Congress and Exposition. Houston, TX.

Deimel, R., \& Brock, O. (2014). A Novel Type of Compliant, Underactuated Robotic Hand for Dexterous Grasping. Robotics: Science and Systems, (pp. 1687-1692). Berkeley, CA.

Diankov, R. (2019). Open Robotics Automation Virtual Environment: An environment for testing, developing, and deploying robotics motion planning algorithms. Retrieved from https://github.com/rdiankov/openrave

Dollar, A. M., \& Howe, R. D. (2010, April). The Highly Adaptive SDM Hand: Design and Performance Evaluation. The International Journal of Robotics Research, 29(5), 585-597.

Edan, Y., \& Miles, G. (1993). Design of an Agricultural Robot for Harvesting Melons. Transactions of the ASAE, 36(2), 593-603.

Edan, Y., \& Miles, G. (1994). Systems Engineering of Agricultural Robot Design. IEEE Transactions on Systems, Man, and Cybernetics, 24(8), 1259-1265.

Edan, Y., Haghighi, K., Stroshine, R., \& Cardenas-Weber, M. (1992, July). Robot Gripper Analysis: Finite Element Modeling and Optimization. Applied Engineering in Agriculture, 8(4), 563-570.

Edan, Y., Rogozin, D., Flash, T., \& Miles, G. (2000, December). Robotic Melon Harvesting. IEEE Transactions on Robotics and Automation, 16(6), 831-834.

Erdogan, D., Guner, M., Dursun, E., \& Gezer, I. (2003). Mechanical Harvesting of Apricots. Biosystems Engineering, 85(1), 19-28.

Feng, Q., Zheng, W., Qiu, Q., Jiang, K., \& Guo, R. (2012). Study on Strawberry Robotic Harvesting System. IEEE International Conference on Computer Science and Automation Engineering (CSAE), (pp. 320-324). Zhangjiajie, China.

Fengying, X., Guolong, L., Xiangjun, Z., Zhen, C., \& Ce, X. (2011). The Virtual Prototype Design and Simulation of Litchi Fruit Flexible Picking Manipulator. 2011 International Conference on Computer Distributed Control and Intelligent Environmental Monitoring. Changsha, China.

Flood, S. J., Burks, T. F., \& Teixeira, A. A. (2006). Physical Properties of Oranges in Response to Applied Gripping Forces for Robotic Harvesting. Transactions of the ASABE, 49(2), 341-346.

Foglia, M. M., \& Reina, G. (2006). Agricultural Robot for Radicchio Harvesting. Journal of Field Robotics, 23(6/7), 363-377.

Galinato, S., \& Gallardo, R. K. (2011). Estimated Cost of Producing Pears in North Central Washington (FS031E). Retrieved January 7, 2013, from http://extecon.wsu.edu/pages/Enterprise_Budgets.

Gallardo, R. K., Taylor, M., \& Hinman, H. (2010). 2009 Cost Estimates of Establishing and Producing Gala Apples in Washington (FS005E). Retrieved January 7, 2013, from http://extecon.wsu.edu/pages/Enterprise_Budgets.

Gongal, A., Amatya, S., Karkee, M., Zhang, Q., \& Lewis, K. (2015). Sensors and Systems for Fruit Detection and Localization: A Review. Computers and Electronics in Agriculture (116), 8-19.

Gonzalez-Barrera, A. (2015). More Mexicans Leaving Than Coming to the U.S. Pew Research Center. Retrieved November 24, 2015, from http:/www.pewhispanic.org/files/2015/11/2015-11-19_mexicanimmigration_FINAL.pdf

Grand D'Esnon, A. (1985). Robotic Harvesting of Apples. Proceedings of Agri-Mation 1. ASAE Paper 1-85. St. Joseph, MI.
Grand D'Esnon, A., Rabatel, G., Pellenc, R., Journeau, A., \& Aldon, M. J. (1987). MAGALI: A Self-Propelled Robot to Pick Apples. ASAE Paper 87-1037. St. Joseph, MI.

Guo, L., Zhan, L., Ping, L., \& Jun, Y. (2009). Study on the CollisionMechanical Properties of Tomatoes Gripped by Harvesting Robot Fingers. African Journal of Biotechnology, 8(24), 7000-7007.

Haifeng, W., Bin, L., Guangyu, L., \& Liming, X. (2012, October). Design and Test of Pineapple Harvesting Manipulator. Transactions of the Chinese Society of Agricultural Engineering, 28(2), 42-46.

Han, K., Kim, S., Lee, Y., Kim, S., Im, D., Choi, H., \& Hwang, H. (2012). Strawberry Harvesting Robot for Bench-type Cultivation. Journal of Biosystems Engineering, 37(1), 65-74.

Harrell, R. C., \& Levi, P. (1988). Vision Controlled Robots for Automatic Harvesting of Citrus. International Conference on Agricultural Engineering, Paper No. 88.426. Paris, France.

Hayashi, A. (1994). Geometric motion planning for highly redundant manipulators using a continuous model. University of Texas at Austin.

Hayashi, S., Shigematsu, K., Yamamoto, S., Kobayashi, K., Kohno, Y., Kamata, J., \& Kurita, M. (2010). Evaluation of a Strawberry-Harvesting Robot in a Field Test. Biosystems Engineering, 105, 160-171.

Hohimer, C., Wang, H., Bhusal, S., Miller, J., Mo, C., \& Karkee, M. (2018). Design and Field Evaluation of a Robotic Apple Harvesting System with a 3D-Printed Soft-Robotic End-Effector. Transactions of the ASABE.

Hwang, H., \& Kim, S.-C. (2003). Development of Multi-functional Teleoperative Modular Robotic System for Greenhouse Watermelon. IEEE/ASME International Conference on Advanced Intelligent Mechatronics (AIM 2003), (pp. 1344-1349). Piscataway, New Jersey.

Irie, N., Taguchi, N., Horie, T., \& Ishimatsu, T. (2009). Asparagus Harvesting Robot Coordinated with 3-D Vision Sensor. IEEE International Conference on Industrial Technology, (pp. 1-6). Gippsland, Australia.

Janisiewicz, W. J., \& Peterson, D. L. (2004). Susceptibility of the Stem Pull Area of Mechanically Harvested Apples to Blue Mold Decay and Its Control with a Biocontrol Agent. Plant Disease, 88(6), 662-664.

Jia, B., Zhu, A., Yang, S., \& Mittal, G. (2009). Integrated Gripper and Cutter in a Mobile Robotic System for Harvesting Greenhouse Products. IEEE International Conference on Robotics and Biomimetics. Guilin, China.

Jimenez, A. R., Ceres, R., \& Pons, J. L. (2000). A Survey of Computer Vision Methods for Locating Fruit on Trees. Transactions of the American Society of Agricultural Engineers, 43(6), 1911-1920.

Jones, D. R., Perttunen, C. D., \& Stuckman, B. E. (1993). Lipschitzian Optimization without the Lipschitz Constant. Journal of Optimization Theory and Applications, 79(1), 157-181.

Kapach, K., Barnea, E., Mairon, R., Edan, Y., \& Ben-Shahar, O. (2012). Computer Vision for Fruit Harvesting Robots - State of the Art and Challenges Ahead. International Journal of Computational Vision and Robotics, 3(1-2), 4-34.

Karkee, M., \& Zhang, Q. (2012, September/October). Mechanization and Automation Technologies in Specialty Crop Production. ASABE Resource Magazine, pp. 16-17.

Kitamura, S., \& Oka, K. (2005). Recognition and Cutting System of Sweet Pepper for Picking Robot in Greenhouse Horticulture. IEEE International Conference on Mechatronics and Automation. Niagara Falls, Canada.

Kondo, N., Nishitsuji, Y., Ling, P., \& Ting, K. (1996). Visual Feedback Guided Robotic Cherry Tomato Harvesting. Transactions of the ASAE, 39(6), 2331-2338.

Koval, M., \& Velgapudi, P. (2016). Or Trajopt Retrieved from https://github.com/personalrobotics/or_trajop

Lee, B. S., \& Rosa, U. A. (2006). Development of a Canopy Volume Reduction Technique for Easy Assessment and Harvesting of Valencia Citrus Fruits. Transactions of the ASABE, 49(6), 1695-1703.

Lehnert, C., Perez, T., \& McCool, C. (2015). Optimisation-based Design of a Manipulator for Harvesting Capsicum. IEEE International Conference on Robotics and Automation (ICRA) Workshop on Robotics in Agriculture. Seattle, WA. 
Li, P., Lee, S., \& Hsu, H. (2011). Review of Fruit Harvesting Method for Potential Use of Automatic Fruit Harvesting Systems. Procedia Engineering, 23, 351-366.

Li, Z., Li, P., \& Liu, J. (2011). Physical and Mechanical Properties of Tomato Fruits as Related to Robot's Harvesting. Journal of Food Engineering, 103, 170-178.

Li, Z., Liu, J., Li, P., \& Li, W. (2008). Analysis of Workspace and Kinematics for a Tomato Harvesting Robot. International Conference on Intelligent Computation Technology and Automation, (pp. 823-827). Changsha, China.

Ling, P. P., Ehsani, R., Ting, K. C., Chi, Y., Ramalingam, N., Klingman, M. H., \& Draper, C. (2004). Sensing and End-Effector for a Robotic Tomato Harvester. ASAE/CSAE International Meeting, Paper 043088, (p. 12 pp). Ottawa, Canada.

Liu, J., Li, P., \& Li, Z. (2007). A Multi-Sensory End-Effector for Spherical Fruit Harvesting Robot. IEEE International Conference on Automation and Logistics. Jinan, China.

Liu, T., Zeng, X., \& Ke, Z. (2011). Design and Prototyping a Harvester for Litchi Picking. Fourth International Conference on Intelligent Computation Technology and Automation, (pp. 39-42). Shenzhen, China.

Monta, M., Kondo, N., \& Shibano, Y. (1995). Agricultural Robot in Grape Production System. International Conference on Robotics and Automation, (pp. 2504-2509). Nagoya, Japan.

Monta, M., Kondo, N., \& Ting, K. C. (1998). End-Effectors for Tomato Harvesting Robot. Artificial Intelligence Review, 12, 11-25.

Muscato, G., Prestifilippo, M., Abbate, N., \& Rizzuto, I. (2005). A Prototype of an Orange Picking Robot: Past History, the New Robot and Experimental Results. Industrial Robot: An International Journal, 32(2), 128-138.

National Agricultural Statistics Service (NASS). (2008-2009). Florida Citrus Statistics. United States Department of Agriculture (USDA). Retrieved from

http://www.nass.usda.gov/Statistics_by_State/Florida/Publications/Citrus/i ndex.asp

OpenRAVE. (2013). Open Robotics Automation Virtual Environment. Retrieved from http://openrave.org/docs/latest_stable/coreapihtml/index.html

Peterson, D. L. (2005, January-March). Harvest Mechanization Progress and Prospects for Fresh Market Quality Deciduous Tree Fruits. HortTechnology, 15(1), 72-75.

Peterson, D. L., \& Wolford, S. D. (2003). Fresh-Market Quality Tree Fruit Harvester Part II: Apples. Applied Engineering in Agriculture, 19(5), 545548.

Peterson, D. L., Miller, S. S., \& Whitney, J. D. (1994). Harvesting Semidwarf Freestanding Apple Trees with an Over-the-Row Mechanical Harvester. Journal of the American Society for Horticultural Science, 119(6), 11141120.

Peterson, D. L., Whiting, M. D., \& Wolford, S. D. (2003). Fresh-Market Quality Tree Fruit Harvester Part I: Sweet Cherry. Applied Engineering in Agriculture, 19(5), 539-543.

Polat, R., Gezer, I., Guner, M., Dursun, E., Erdogan, D., \& Bilim, H. (2007). Mechanical Harvesting of Pistachio Nuts. Journal of Food Engineering, 79, 1131-1135.

Pool, T. A., \& Harrell, R. C. (1991, March-April). An End-Effector for Robotic Removal of Citrus from the Tree. Transactions of the ASAE, 34(2), 373378.

Reed, J. N., Miles, S. J., Butler, J., Baldwin, M., \& Noble, R. (2001). Automatic Mushroom Harvester Development. Journal of Agricultural Engineering Research, 78(1), 15-23.

Rodriguez, F., Moreno, J., Sanchez, J., \& Berenguel, M. (2013). Grasping in Agriculture: State-of-the-Art and Main Characteristics. In Grasping in Robotics (pp. 385-409). London: Springer.

Rus, D., \& Tolley, M. (2015, May). Design, Fabrication, and Control of Soft Robots. Nature, 521, 467-475.
Sakai, S., Iida, M., Osuka, K., \& Umeda, M. (2008). Design and Control of a Heavy Material Handling Manipulator for Agricultural Robots. Autonomous Robots, 25(3), 189-204.

Sanders, K. F. (2005). Orange Harvesting Systems Review. Biosystems Engineering, 90(2), 115-125.

Sarig, Y. (1993). Robotics of Fruit Harvesting: A State-of-the-art Review. Journal of Agricultural Engineering Research, 54, 265-280.

Scarfe, A. J., Flemmer, R. C., Bakker, H. H., \& Flemmer, C. L. (2009). Development of an Autonomous Kiwifruit Picking Robot. $4^{\text {th }}$ International Conference on Autonomous Robots and Agents. Wellington, New Zealand.

Schmid, K., Lutz, P., Tomic, T., Mair, E., \& Hirschmuller, H. (2014). Autonomous Vision-based Micro Air Vehicle for Indoor and Outdoor Navigation. Journal of Field Robotics, 31(4), 537-570.

Schulman, J., Ho, J., Lee, A., Awwal, I., Bradlow, H., \& Abbeel, P. (2013). Finding Locally Optimal, Collision-Free Trajectories with Sequential Convex Optimization. Robotics: Science and Systems Foundation.

Setiawan, A. I., Furukawa, T., \& Preston, A. (2004). A Low-Cost Gripper for an Apple Picking Robot. IEEE International Conference on Robotics and Automation. New Orleans, Louisiana.

Shamshiri, R., Hameed, I., Karkee, M., \& Weltzien, C. (2018d). Robotic Harvesting of Fruiting Vegetables: A Simulation Approach in V-REP, ROS and MATLAB. In R. Shamshiri, I. Hameed, M. Karkee, \& C. Weltzien, Automation in Agriculture - Securing Food Supplies for Future Generations.

Shamshiri, R., Hameed, I., Pitonakova, L., Weltzien, C., Balasundram, S., Yule, I., et al. (2018c). Simulation software and virtual environments for acceleration of agricultural robotics: Features highlights and performance comparison. International Journal of Agricultural and Biological Engineering, 11(4), 15-31.

Shamshiri, R., Kalantari, F., Ting, K., Thorp, K., Hameed, I., Weltzien, C., et al. (2018b). Advances in greenhouse automation and controlled environment agriculture: A transition to plant factories and urban agriculture. International Journal of Agricultural and Biological Engineering, 11(1), 1-22.

Shamshiri, R., Weltzien, C., Hameed, I., Yule, I., Grift, T., Balasundram, S., et al. (2018a). Research and development in agricultural robotics: A perspective of digital farming. International Journal of Agricultural and Biological Engineering 11(4), 1-14.

Silwal, A., Davidson, J., Karkee, M., Mo, C., Zhang, Q., \& Lewis, K. (2017). Design, integration, and field evaluation of a robotic apple harvester. Journal of Field Robotics 34(6), 1140-1159.

Song, H., Sun, X., Zhang, T., Zhang, B., \& Xu, L. (2007). Design Optimisation and Simulation of Structure Parameters of an Eggplant Picking Robot. New Zealand Journal of Agricultural Research, 50(5), 959-964.

Soria, P., Sukkar, F., Martens, W., Arrue, B., Fitch, R., Ollero, A., et al. (2018). Multi-view probabilistic segmentation of pome fruit with a lowcost RGB-D camera. ROBOT 2017: Third Iberian Robotics Conference (pp. 320-331).

Sukkar, F. (2018). Fast, reliable and efficient database search motion planner (FREDS-MP) for repetitive manipulator tasks. University of Technology Sydney.

Sun, Q., Zou, X., Zou, H., Chen, Y., \& Cai, W. (2010). Intelligent Design and Kinematics Analysis of Picking Robot Manipulator. International Conference on Measuring Technology and Mechatronics Automation, (pp. 493-496). Changsha, China.

Tang, X., Zhang, T., Liu, L., Xiao, D., \& Chen, Y. (2009). A New Robot System for Harvesting Cucumber. ASABE International Meeting, (pp. 3873-3885). Reno, Nevada.

Tanigaki, K., Fujiura, T., Akase, A., \& Imagawa, J. (2008). Cherry-Harvesting Robot. Computers and Electronics in Agriculture, 63, 65-72.

Tong, J., Zhang, Q., Karkee, M., Jiang, H., \& Zhou, J. (2014). Understanding the Dynamics of Hand Picking Patterns of Fresh Market Apples. ASABE and CSBE/SCGAB Annual International Meeting, (p. 6 pp). Montreal, Canada. 
Torregrosa, A., Orti, E., Martin, B., Gil, J., \& Ortiz, C. (2009). Mechanical Harvesting of Oranges and Mandarins in Spain. Biosystems Engineering, 104, 18-24.

Umeda, M., Kubota, S., \& Iida, M. (1999). Development of "STORK", a Watermelon-Harvesting Robot. Artificial Life Robotics, 3, 143-147.

USDA. (2002, December 19). United States Standards for Grades of Apples. Retrieved October 10, 2015, from

http://www.ams.usda.gov/sites/default/files/media/Apple_Grade_Standard $\% 5 \mathrm{~B} 1 \% 5 \mathrm{D} . \mathrm{pdf}$

USDA. (2008). USDA Agricultural Marketing Service. Retrieved from Definition of specialty crops: http://www.ams.usda.gov/AMSv1.0/scbgpdefinitions

Van Henten, E. J., Hemming, J., Van Tuijl, B., Kornet, J. G., Meuleman, J., Bontsema, J., \& Van Os, E. A. (2002). An Autonomous Robot for Harvesting Cucumbers in Greenhouses. Autonomous Robots, 13, 241-258.

Van Henten, E. J., Van Tuijl, B. J., Hemming, J., Kornet, J. G., Bontsema, J., \& Van Os, E. A. (2003). Field Test of an Autonomous Cucumber Picking Robot. Biosystems Engineering, 86(3), 305-313.

Van Henten, E. J., Van't Slot, D. A., Hol, C. J., \& Van Willigenburg, L. G. (2009). Optimal Manipulator Design for a Cucumber Harvesting Robot. Computers and Electronics in Agriculture, 65, 247-257.
Wan Ishak, W., Kit, W., \& Awal, M. (2010). Design and Development of Eggplant Harvester for Gantry System. Pertanika J. Sci. \& Technol., 18(2), 231-242.

Wang, H., Hohimer, C., Bhusal, S., Karkee, M., Mo, C., \& Miller, J. (2018). Simulation As A Tool In Designing And Evaluating A Robotic Apple Harvesting System. IFAC-PapersOnLine, 51(17), 135-140.

Wehner, M., Tolley, M., Menguc, Y., Park, Y., Mozeika, A., Ding, Y., et al. (2014). Pneumatic Energy Sources for Autonomous and Wearable Soft Robotics. Soft Robotics, 2, 1-12.

Yang, D. C., \& Lee, T. W. (1984). Heuristic Combinatorial Optimization in the Design of Manipulator Workspace. IEEE Transactions on Systems, Man, and Cybernetics, 14(4), 571-580.

Yoshikawa, T. (1985). Manipulability of Robotic Mechanisms. International Journal of Robotics Research, 4(2), 3-9.

Zhang, P., Yao, Z., \& Du, Z. (2014). Global Performance Index System for Kinematic Optimization of Robotic Mechanism. Journal of Mechanical Design, 136(3), 11 pgs.

Zhao, D., Lu, J., Ji, W., Zhang, Y., \& Chen, Y. (2011). Design and Control of an Apple Harvesting Robot. Biosystems Engineering, 110, 112-122.

Zhao, Y., Gong, L., Liu, C., \& Huang, Y. (2016). Dual-arm Robot Design and Testing for Harvesting Tomato in Greenhouse. IFAC-PapersOnLine. 